\title{
STUDIES OF IODOALBUMIN METABOLISM. I. A MATHEMATICAL APPROACH TO THE KINETICS *
}

\author{
By CHARLES G. LEWALLEN, $\uparrow$ MONES BERMAN, $\dagger$ AND JOSEPH E. RALL $\dagger$ \\ (From the Medical Department, Brookhaven National Laboratory, Upton, N. Y.; the Division of Biophysics, \\ Sloan-Kettering Institute, Memorial Center, New York, N. Y.; and the National Institute of \\ Arthritis and Metabolic Diseases, National Institutes of Health, Bethesda, Md.)
}

(Submitted for publication May 21, 1958; accepted September 18, 1958)

During the clinical study and treatment of a group of patients with thyroid carcinoma, we have studied the effects of thyroid hormone on several metabolic systems under conditions in which the thyroid status of the patients could be controlled at will. Early in the tracer studies of iodoalbumin metabolism in these patients it was apparent that it was necessary to employ a mathematical approach somewhat different from published methods of compartment analysis. Accordingly a suitable mathematical approach was developed by one of the present authors (1). In this report, the practical difficulties encountered in application of the approach to experimental data and the theoretical implications of the approach are discussed.

The kinetic analysis of the data of a single tracer study comprises the body of the report. Additional data from other tracer studies are included only insofar as they contribute to the development of the model chosen for analysis.

\section{METHODS}

General. The methods of dialysis of the commercial $I^{131}$ labeled human serum albumin employed and details of the injection procedure, collection of blood and urine samples, quantitation of radioactivity in urine and plasma, determination of serum albumin and quantitation of total radioactivity removed in blood sampling have been given elsewhere (2). Stool collections were made only in the case of M. H. in Experiment III. The individual specimens were suspended in concentrated sodium hydroxide and distilled water to known volume, allowed to digest partly, and then agitated until a uniform pipettable mixture was obtained. The counting procedure was the same as that for urine and plasma (2). None of the patients studied showed albuminuria No chemical fractionation of label was done routinely on urine or plasma samples.

* This work was supported in part by the Atomic Energy Commission.

† Present address: National Institute of Arthritis and Metabolic Diseases, National Institutes of Health, Bethesda, Md.
In an ancillary study, Patient G. S., hospitalized because of breast carcinoma with bone metastases, but clinically euthyroid and without evidence of cardiac, renal or hepatic disease, was given $2 \mathrm{mc}$. of iodoalbumin intravenously and two 24 hour urine collections were made. Chemical fractionation with added carrier of the first urine showed 0.004 of the label precipitable with 5 per cent trichloracetic acid, 0.854 precipitable with 10 per cent silver nitrate, and 0.144 not precipitable with either agent. The respective figures for the second 24 hour urine were $0.004,0.886$ and 0.108 . Paper chromatography showed the major constituents to be iodide (about 90 per cent) and diiodotyrosine (about 10 per cent). In the case of M. H., Experiment III, serum samples taken on Days 4 and 8 were subjected to paper electrophoresis. Virtually all of the label could be accounted for in the albumin fraction.

Selection of patients and description of experiments. The two experiments reported here (I and III) were carried out on five patients, all under observation for treatment of histologically proven thyroid carcinoma. All had previously had either surgical removal or $I^{131}$ radiation destruction of their thyroids or both as part of their therapeutic regimen. Those patients developing severe hypothyroidism or myxedema were chosen for the iodoalbumin tracer studies.

Additional details of the clinical status of the patients and the experimental protocol for Experiment I are given in Reference (2). Features relevant to the present report follow.

Experiment $I$. This experiment was performed to study the metabolism of iodoalbumin in the euthyroid and myxedematous steady states in the same individual. Four patients were studied, each in both states on different occasions. Blood and urine samples were collected for 14 days following injection of the $\mathrm{I}^{131}$-albumin. At the time of the first tracer study all had gross clinical evidence of myxedema. Otherwise they were fully ambulatory and in good general physical condition throughout both studies. In six studies the thyroid uptake of $I^{131}$ was blocked either by an antithyroid drug or following cholecystography. In the two studies carried out without blocking agents the patients demonstrated their inability to retain ${ }^{131}$ presented as iodide ion by excreting in four days 96 and 100 per cent, respectively, of the label administered as $\mathrm{NaI}^{131}$ in tracer studies carried out subsequent to the iodoalbumin studies but under the same metabolic conditions.

Experiment III. This experiment was performed for four reasons: 1) to quantitate fecal excretion of label. 
2) to follow urinary excretion and plasma levels for a prolonged period during a steady metabolic state, 3) to study in a recipient subject the metabolic behavior of iodoalbumin previously subjected to an eight day tenure in a donor subject, and 4) to study carefully the early renal excretion of label in the recipient. It was thought that the donor would act as a biological "filter" and remove from the labeled material the more rapidly degraded constituents, if any were present, rendering a more homogeneous product for the recipient tracer study.

Two patients were studied. The donor, M. H., was an 81 year old female with known thyroid cancer and cranial metastases for nine years. She had received multiple $\mathrm{I}^{131}$ therapies in the previous four years with almost complete destruction of the cranial lesion but with persistence of a large cervical tumor. In addition she had hypertension and arteriosclerotic heart disease with angina pectoris but no evidence of congestive failure or hepatic or renal disease. Two months prior to the experiment her cholesterol was $364 \mathrm{mg}$. per cent and she was started on Tapazole ${ }^{\circledR}$, $40 \mathrm{mg}$. daily. The latter was continued throughout the 63 days of the study during which time she exhibited evidence of myxedema. During the study her mean cholesterol was $328 \mathrm{mg}$. per cent; her mean basal metabolic rate (BMR), -37 per cent. Her weight varied from $65.1 \mathrm{Kg}$. at the beginning to $64.5 \mathrm{Kg}$. at the end of the study. A NaI ${ }^{131}$ tracer given after completion of the study showed a urinary excretion of 0.966 of the dose in 10 days.

The pertinent clinical data on R. B., the recipient, appear elsewhere (2). For one month prior to and during the study she received Tapazole ${ }^{\circledR}, 40 \mathrm{mg}$., and triiodothyronine, $150 \mu \mathrm{g}$., daily. She was also given saturated solution of potassium iodide, 15 minims daily, during the experiment. Her weight varied between 49.1 and 50.6 $\mathrm{Kg}$. Her electrocardiogram was normal, her mean cholesterol $149 \mathrm{mg}$. per cent. Clinically she appeared mildly hyperthyroid.

On Day 1 of the study, M. H., the donor, was given intravenously $4.82 \mathrm{mc}$. of $\mathrm{I}^{131}$ iodoalbumin. Plasma samples were taken as previously described. All urinary voidings were collected separately for the first six days, after which 24 hour collections were made. On Day $8,450 \mathrm{ml}$. of blood representing $281 \mathrm{ml}$. of plasma was taken by venepuncture under sterile precautions with simultaneous replacement transfusion. (The latter was considered mandatory in view of the donor's cardiovascular status.) The plasma was separated by centrifugation and three hours after venesection $240 \mathrm{ml}$. of the nondialyzed donor plasma of activity $37 \mu \mathrm{c}$. was given intravenously to R. B. The injection required 28 minutes. The first plasma sample was taken six minutes following completion of the injection.

All urinary voidings were collected separately for the first six days and then 24 hour collections were made. In the case of $\mathrm{R}$. B. the study was continued for 20 days following injection. An intravenous $\mathrm{NaI}^{131}$ tracer performed after completion of the iodoalbumin study showed 0.966 urinary excretion of $I^{131}$ in four days. Similarly an intravenous tracer of $\mathrm{I}^{131}$-diiodotyrosine under the same regimen that existed during the iodoalbumin study showed 0.994 urinary excretion of $\mathrm{I}^{131}$ in five days.
In the case of R. B. the per cent probable error of counting of the least active plasma samples did not exceed 0.9 per cent; that of the least active urine samples, 3.4 per cent. For M. H. the respective figures were 0.8 and 1.2 per cent. For the stool samples of M. H. the per cent probable error did not exceed 6.4 per cent except in 14 samples toward the end of the study where the probable error was as high as 15 per cent.

Units and graphical representation of data. Plasma specific activity was expressed as fraction of the injected dose of radioactivity per gram of albumin. In Experiments I and III the variation in serially determined plasma albumin concentrations was within the error of the method.

Plasma specific activity was plotted against time (in hours) on semilogarithmic coordinates. In all cases these curves could be reduced to a minimum of three exponential components by standard methods of graphical analysis. The slowest component was obtained first by a least squares fit of the terminal linear portion of the plot. The remainder of the curve was smoothed by eye, and the two additional components were obtained by serial subtraction in the usual way (3). The standard errors of the slope and of the ordinate intercept of the slowest component were computed according to the method described by Snedecor (4). The exponential constant, $\alpha$, for the slow component was available from the least squares calculations. The $T_{1}$ of each of the faster components was obtained directly from the graph and the exponential constant computed from the relationship: $\alpha \mathrm{T}_{\frac{1}{3}}=0.693$. The ordinate intercepts of the three components gave the coefficients, $\mathrm{X}_{\mathrm{ji}}$, of the exponential terms.

Urinary excretion data were plotted as cumulative fraction retained (1-cumulative excretion)' on semilogarithmic coordinates. The cumulative fraction retained was corrected for fraction removed in blood sampling in Experiment III. In the case of M. H. the total fraction of the injected dose removed in blood sampling over the 63 days of the experiment was 0.0347 , including 0.0177 removed to obtain labeled plasma for injection into R. B. In the case of R. B., Experiment III, 0.025 of the injected dose was removed in blood sampling over a 20 day period (larger blood samples were required because of the relatively small amount of radioactivity injected). In all other instances the fraction removed in sampling was less than 0.01 and was disregarded. In the case of M. H., Experiment III, the cumulative fecal excretion in 63 days was 0.0156 . This was taken into account in computing the cumulative fraction retained in this instance, but fecal excretion was not measured and was disregarded in all of the other studies, which were of shorter duration.

The terminal, apparently linear, portions of the retention curves (beginning about the fourth or fifth day) were fitted by the method of least squares and the standard errors of the slopes and ordinate intercepts computed by the same approach that was used for the plasma curves.

\section{RESULTS AND ANALYSIS OF DATA}

\section{Definitions}

Carrier: The unlabeled form of the substance being studied. 
Compartment: An aggregate of molecules which have the same transition probability from the state they are in to all other available states.

Iodoalbumin degradation: Alteration of the iodoalbumin molecule such that iodine in an excretable form is liberated.

Three-compartment series model: A threecompartment system in which the originally labeled compartment interacts with only one of the two remaining compartments.

Three-compartment parallel model: A threecompartment system in which the originally labeled compartment interacts with both the remaining compartments which in turn do not interact with each other.

$A_{j i}$ : The coefficient of the $i^{\text {th }}$ exponential term in the equation describing the fraction of injected tracer in the $j^{\text {th }}$ compartment as a function of time. Graphically, an ordinate intercept.

$\alpha_{\mathrm{i}}$ : Exponential constant (fraction per hour) in the $\mathrm{i}^{\text {th }}$ exponential term.

$S_{j}$ : The amount of carrier albumin (grams) in the $\mathbf{j}^{\text {th }}$ compartment.

$\mathrm{S}_{\mathrm{T}}$ : Sum of carrier (grams) in all compartments (total exchangeable albumin).

$q_{j}(t)$ : Quantity of label (fraction of injected dose) in compartment $j$ at time $t$.

$\mathbf{x}_{\mathbf{j}}(\mathrm{t})$ : Specific activity (fraction of injected dose of label per gram of carrier) of compartment $\mathrm{j}$ at time $\mathrm{t}$.

$X_{j i}$ : Coefficient of the $i^{\text {th }}$ exponential term in the equation describing the specific activity of the $j^{\text {th }}$ compartment as a function of time. Graphically an ordinate intercept.

$\lambda_{i j}$ : Fraction of $S_{j}$ or $q_{j}(t)$ transferred to compartment $\mathrm{i}$ from compartment $\mathrm{j}$ per hour; $\mathrm{a}$ fractional turnover rate. ${ }^{1}$

$\lambda_{o j}$ : Fraction of $S_{j}$ or $q_{j}(t)$ leaving the exchanging system per hour by all avenues of unidirectional loss. ${ }^{1,2}$

$\lambda_{j j}$ : Sum of all fractional rates of loss of $S_{j}$ or $q_{j}(t)$ from compartment $j$ per hour; the total fractional turnover rate of compartment $j$.

\footnotetext{
${ }^{1}$ We wish to emphasize that the subscripts of $\lambda_{i j}$ and $\rho_{i j}$ are read "to $i$ from $j . "$ This is a reversal of the directional sense of the subscript notation commonly appearing in the biological literature and was employed here because of greater convenience in matrix operations and to preserve notational consistency with Reference (1).

2 Unidirectional loss, as used here, includes such processes as iodoalbumin degradation, proteinuria and transfer to nonexchanging compartments.
}

$\lambda_{\mathrm{OT}}$ : Fraction of $S_{\mathrm{T}}$ leaving the exchanging system per hour by all avenues of unidirectional loss. ${ }^{1,2}$

$\rho_{\mathrm{ij}}$ : Rate of transfer of carrier (grams per hour) to compartment $i$ from compartment $j .{ }^{1}$

$\rho_{\mathrm{s}}$ : Steady state rate of synthesis of carrier (grams per hour).

$\mathrm{K}_{\mathrm{ie}}$ : Fraction of iodide in the iodide space excreted by the kidneys per hour.

$\mathrm{K}_{\mathrm{de}}$ : Fraction of diiodotyrosine in the diiodotyrosine space excreted by the kidneys per hour.

\section{Development of a schematic model for kinetic analy-} sis of data

Evidence has been presented by many investigators for the existence of an extravascular albumin compartment approximating in size the plasma albumin (5-7). The model proposed in this paper consists of plasma (Compartment 1), two extravascular exchanging compartments (Compartments 2 and 3), a compartment containing nonexcreted degradation products (Compartment 4), collected urine (Compartment 5) and an extravascular nonexchanging compartment (Compartment 6). Several arguments to justify the choice of this model follow.

The data of R. B., Experiment III, are presented graphically in Figure 1. Graphic analysis of the plasma curves indicated that in all the tracer studies plasma specific activity could be expressed as a function of time by an equation involving a minimum of three exponential terms:

1) $\mathrm{x}_{1}(\mathrm{t})=\mathrm{X}_{11} \mathrm{e}^{-\alpha_{1} \mathrm{t}}+\mathrm{X}_{12} \mathrm{e}^{-\alpha_{2} t}+\mathrm{X}_{13} \mathrm{e}^{-\alpha_{3} t}$.

The fact that three exponential components were necessary to fit the plasma curves requires that the system be composed of at least three compartments (1). The recovery of label in urine and feces indicates a system of the open type.

It can be shown (1) for a three-compartment steady state system, open or closed, that the specific activity or quantity of labeled material in each of the compartments may be expressed as a function of time by an equation involving three exponential terms, the exponential constants of which $\left(\alpha_{i}\right)$ are identical for all the compartments:

$$
\begin{gathered}
\mathrm{q}_{1}(\mathrm{t})=\mathrm{A}_{11} \mathrm{e}^{-\alpha_{1} t}+\mathrm{A}_{12} \mathrm{e}^{-\alpha_{2} t}+\mathrm{A}_{13} \mathrm{e}^{-\alpha_{3} t} \\
\text { where } \\
\sum_{\mathrm{i}} \mathrm{A}_{1 \mathrm{i}}=1
\end{gathered}
$$




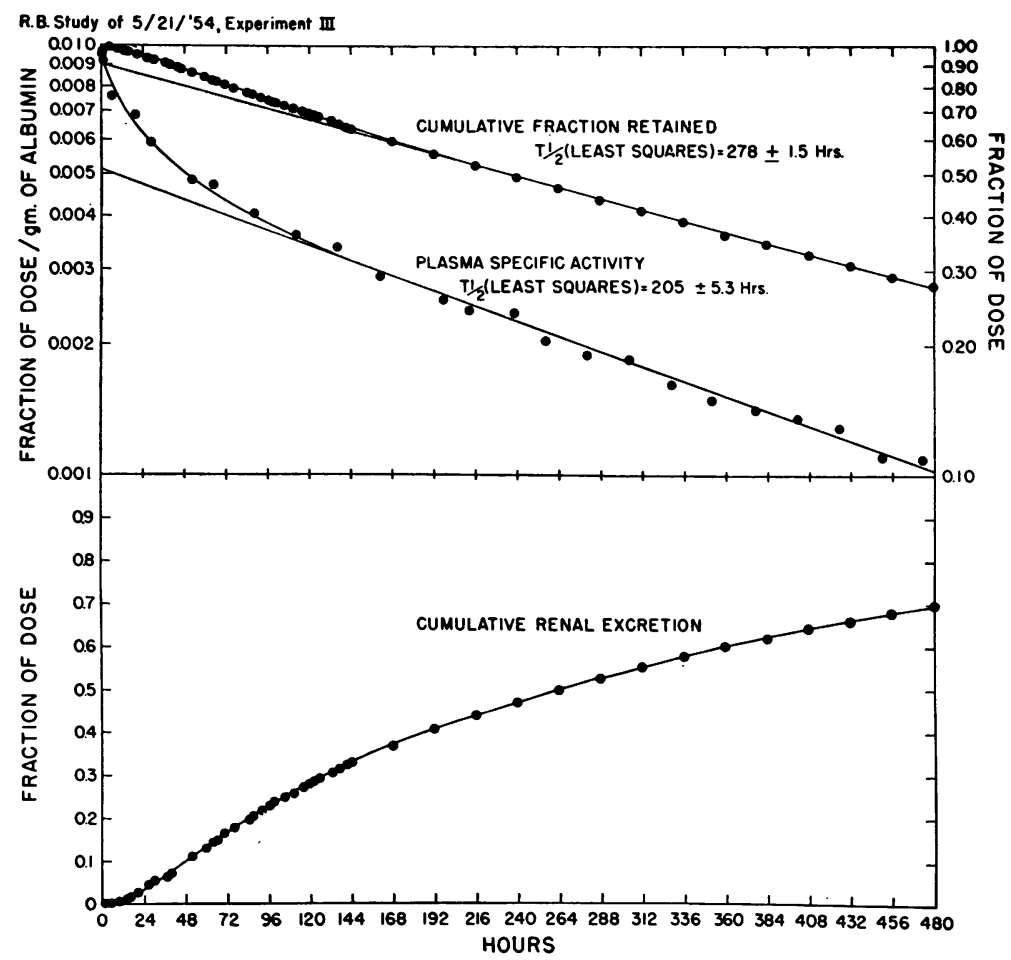

Fig. 1. Typical Plasma and Excretion Data Obtained in Tracer Studies OF IODOALBUMin METABolism linear.

The coordinates of the upper two curves are semilogarithmic; those of the lower curve are

b) $\mathrm{q}_{2}(\mathrm{t})=\mathrm{A}_{21} \mathrm{e}^{-\alpha_{1} \mathrm{t}}+\mathrm{A}_{22} \mathrm{e}^{-\alpha_{2} \mathrm{t}}+\mathrm{A}_{23} \mathrm{e}^{-\alpha_{3} \mathrm{t}}$, where

$$
\sum_{i} A_{2 i}=0
$$

c) $\mathrm{q}_{3}(\mathrm{t})=\mathrm{A}_{31} \mathrm{e}^{-\alpha_{1} t}+\mathrm{A}_{32} \mathrm{e}^{-\alpha_{2} \mathrm{t}}+\mathrm{A}_{33} \mathrm{e}^{-\alpha_{3} \mathrm{t}}$, where

$$
\sum_{\mathbf{i}} A_{3 i}=0
$$

Equation 1 may be normalized to Equation $2 a$ by setting

$$
\text { 3) } \quad \mathrm{A}_{1 \mathrm{i}}=\frac{\mathrm{X}_{1 \mathrm{i}}}{\mathrm{X}_{11}+\mathrm{X}_{12}+\mathrm{X}_{13}} \text {. }
$$

It follows, therefore, that experimental data obtained on the amount or specific activity of isotope in any one compartment or any linear combination of compartments should yield (within experimental error) the same set of slopes ( $\alpha$ 's) when analyzed as a sum of exponential terms.

Comparison of the retention curve and plasma specific activity curve in Figure 1 shows that both approach straight lines after about 120 hours. The terminal slopes of the lines are significantly different, however, with plasma having the greater slope. A similar significant discrepancy in terminal plasma and retention slopes measured over approximately the same time interval was encountered in all the tracer studies performed. From what has been said above, if the retention curve represented the sum of the exchanging albumin compartments, its final slope would have been the same as the final plasma slope. The slower slope of the retention curve suggests that it includes an additional compartment having negligible feedback to the exchanging system during the experiment. A similar discrepancy in slopes was noted in some but not all instances by Berson, Yalow, Schreiber and Post and was attributed to incomplete urine collections (7). Comparable findings are also evident in Myant's data in connection with studies of $\mathrm{I}^{131}$ gamma globulin (8). Further support for the loss of label from the exchanging system to a compartment other than urine comes from the fact that the asymptotic value of cumu- 
lative urinary excretion was well below 100 per cent of the dose. Although measurements were not carried out long enough to determine the value of the asymptote experimentally, it can be computed as follows:

With $\mathrm{q}_{5}(\mathrm{t})$ defined as the cumulative urinary excretion of label, a plot of the logarithm of the daily urinary excretion, $\frac{\mathrm{dq}_{\mathrm{s}}(\mathrm{t})}{\mathrm{dt}}$, shows that its terminal slope $\left(\alpha_{3}\right)$ is virtually the same as the terminal slope of the plasma curve. If, at time $t$, the terminal slope of $\frac{\mathrm{dq}_{\mathrm{s}}(\mathrm{t})}{\mathrm{dt}}$ has become established and if it is assumed that the terminal slope will remain constant indefinitely, one can compute the total expected excretion after time $t_{1}$ :

4) $\quad \int_{t_{1}}^{\infty}\left[\frac{\mathrm{dq}_{5}(\mathrm{t})}{\mathrm{dt}}\right] \mathrm{dt}=\left[\frac{\mathrm{dq}_{5}\left(\mathrm{t}_{1}\right)}{\mathrm{dt}}\right] \frac{1}{\alpha_{3}}$,

where $\frac{d q_{5}\left(t_{1}\right)}{d t}$ is the rate of urinary excretion of label at time $t_{1}$. If the cumulative quantity of label in the urine at time $t_{1}$ is $q_{5}\left(t_{1}\right)$, the asymptotic value of urinary excretion $\left(A_{55}\right)$ becomes:

5) $\mathrm{q}_{5}(\infty) \equiv \mathrm{A}_{55}=\mathrm{q}_{5}\left(\mathrm{t}_{1}\right)+\left[\frac{\mathrm{dq}_{5}\left(\mathrm{t}_{1}\right)}{\mathrm{dt}}\right] \frac{1}{\alpha_{3}}$.

In all the tracer studies performed, the value of $A_{b b}$ so computed was less than unity. The mean value obtained in 11 studies was 0.807 . The difference of 0.193 must represent loss from the system into a site other than collected urine.

These findings indicated that even after a reasonable period for mixing, the label continues to leave the plasma at a faster rate than can be accounted for by collected urinary excretion and sampling. Among the more likely possibilities are the following:

1) Significant external loss of label by routes other than renal, or incomplete urinary collections. This seems unlikely in view of the demonstrated negligible fecal excretion and the recovery in urine of administered iodide $\mathrm{I}^{181}$ and diiodotyrosine $\mathrm{I}^{131}$ in amounts closely approaching 100 per cent of the administered dose. Label removed in sampling was quantitated and corrected for when it exceeded 1 per cent of the dose.

2) Entry of label into the thyroidal system with resultant delay in excretion. This is unlikely in view of the negligible fecal excretion and virtually quantitative urinary recovery of $I^{181}$ administered as sodium iodide, verifying the adequacy of blockage of I $^{131}$ uptake of any functioning thyroidal tissue present.

3) Loss of label to an extravascular compartment (exclusive of the thyroid) having a very slow turnover rate.

In this paper the third possibility is accepted

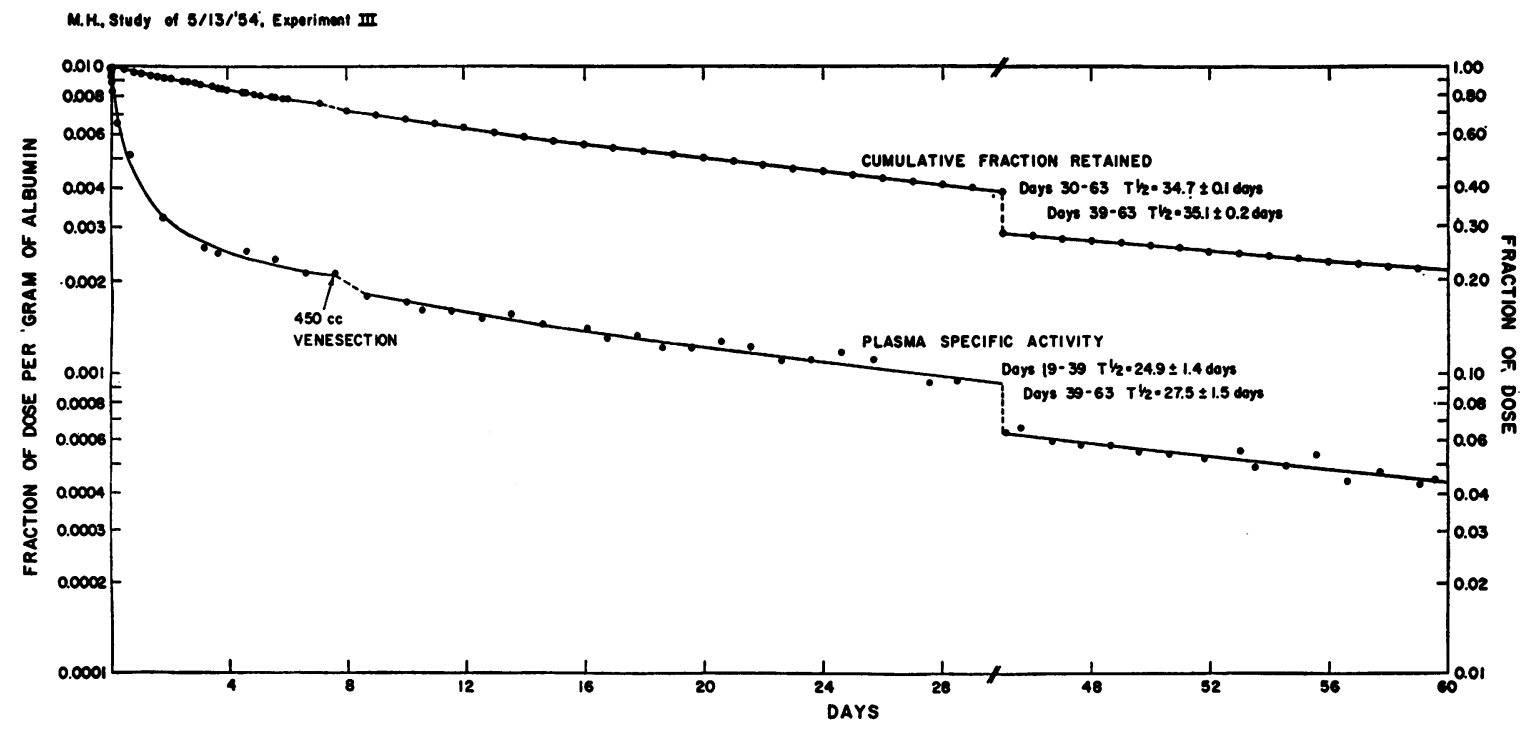

Fig. 2. Metabolism of Imil Albumin in Patient M. H.

Part of the plot has been omitted to facilitate presentation. 
and the presence of an extravascular compartment showing negligible feedback of label to plasma during the course of the experiments is postulated. We wish to emphasize that the experimental evidence for the existence of this postulated Compartment 6 rests entirely on the fact that the calculated values for asymptotic urinary excretion were less than 100 per cent of the injected dose.

If an extravascular nonthyroidal compartment not readily in exchange with plasma exists, the question of its rate of turnover arises. After a sufficiently long time lapse, one would expect the final slopes of radioactivity in each compartment and the slope of their sum, the total label retention, to become equal to the slope of the plasma curve. Equality of plasma and retention slopes was not reached in studies carried out to 36 days [Experiment II, (2)]. For this reason fecal and urinary excretion of label, plasma specific activity and label removed in blood sampling were measured in the study of M. H., Experiment III, for 63 days.

The data are plotted in Figure 2. The breaks in the plasma specific activity and retention curves associated with the withdrawal and transfusion of blood on the eighth day are easily seen. The relative slowness with which the retention and plasma curves approach an apparent constant final slope might be explained by a nonhomogeneous population of injected iodoalbumin containing some elements more rapidly degraded than others or by the presence in the transfused blood of an agent capable of accelerating degradation.

In any event, the retention curve at about 30 days and the plasma curve at about 19 days closely approach an apparent constant final slope. The $T_{1}$ obtained by a least squares fit of the plasma curve from the nineteenth to thirty-ninth days is $24.9 \pm 1.4$ days, and from the thirtyninth to sixty-third days, $27.5 \pm 1.5$ days. From the thirty-ninth to sixty-third days the $T_{\frac{1}{1}}$ of the retention curves is $35.1 \pm 0.2$ days. The difference in slope of the plasma and retention curves (thirty-ninth to sixty-third days) is highly significant statistically. The two curves show little tendency to become parallel. It is apparent that, in this patient, to achieve equality of slopes would require a period considerably longer than 63 days.

Tracer experiments of sufficient duration to allow quantitation of $S_{T}$ (including the postulated slowly exchanging Compartment 6) appeared impractical. It seemed more reasonable to treat Compartment 6 analytically, not as an exchanging compartment, but as an internal, nonfeedback compartment.

Knowledge relative to the mechanism and site of iodoalbumin degradation is limited. Berson and Yalow (9) noted an apparent closer correlation between plasma radioactivity and renal excretion than between radioactivity in ascitic fluid or extravascular space and renal excretion, from which the major site of iodoalbumin degradation was inferred to be the plasma or some extravascular compartment equilibrating rapidly with plasma. The urine fractionation carried out in the case of G. S. (see "Methods") indicated that the excretion forms of label were principally iodide ion and diiodotyrosine.

It has been shown by Albert and Keating (10) and confirmed in this study that in the presence of adequate thyroid block, the radioactivity of labeled iodide ion or diiodotyrosine given intravenously is recovered virtually quantitatively in the urine. Similarly Skanse (11) reported a 72 hour urinary recovery of 98 per cent of $I^{131}$ administered to thyroidectomized patients. This suggests that once the breakdown of iodoalbumin has progressed to the stage of iodide or iodinated tyrosines, the products of breakdown are quantitatively excreted in the urine. Degradation, subsequent to this stage, can reasonably be regarded as a nonfeedback process without reincorporation of iodine into protein.

The volume of distribution of iodide and its rate constant of excretion have been extensively studied. Representative figures from the literature give the volume of distribution of iodide as approximately 34 per cent of the body weight and the rate constant of iodide excretion as $\mathbf{0 . 0 7 2}$ per hour (12). Though diiodotyrosine is, for the most part, rapidly deiodinated when given intravenously and excreted principally as iodide, Albert and Keating (10) calculated the rate constant of excretion of diiodotyrosine per se as $\mathbf{0 . 0 1 3}$ per hour.

It is clear that once label is liberated from 


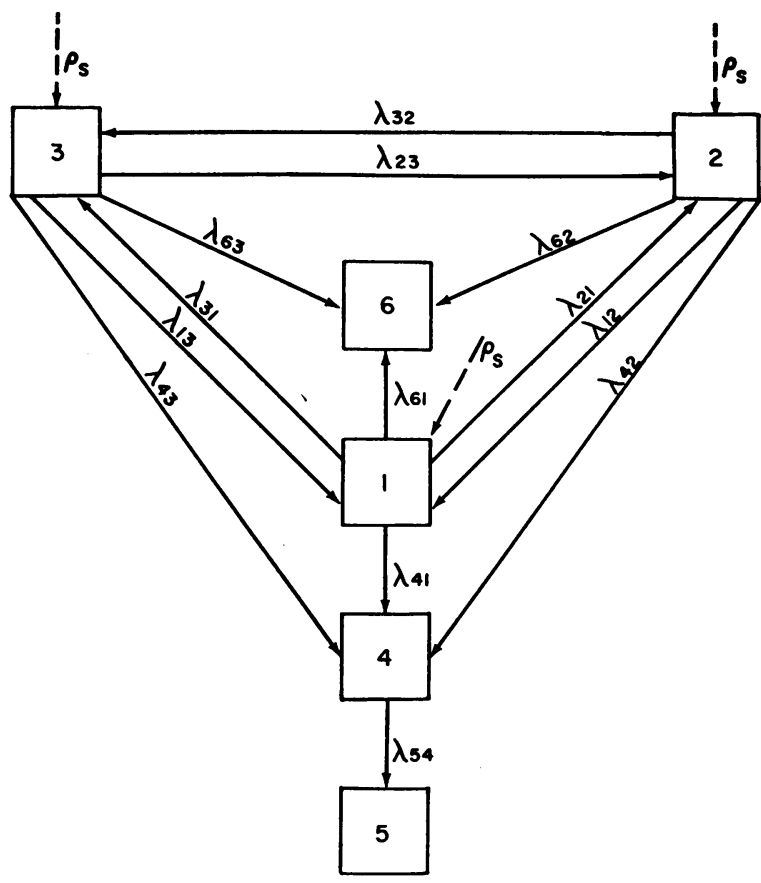

Fig. 3. A Six-Compartment System Consisting of Three Exchanging Compartments (1, 2 and 3) and Three Ancillary Nonexchanging Compartments (4, 5 AND 6)

Compartment 1 (plasma) is the initially labeled and sampled compartment. Compartments 2 and 3 are, respectively, rapidly and slowly exchanging extravascular compartments. Compartment 6 is the postulated body compartment treated analytically as a nonexchanging compartment. Compartment 4 represents nonexcreted labeled degradation products. Compartment 5 is collected urine. The entry site of newly synthesized carrier is not restricted and is depicted (broken arrows) as being either of the exchanging compartments. The rate constants of transfer (the fractional turnover rates) and the steady state rate of carrier synthesis $\left(\rho_{\mathrm{B}}\right)$ appear beside the appropriately directed arrows.

iodoalbumin, it is not instantaneously excreted. Furthermore, the volumes of distribution of iodoalbumin and iodide are markedly different, comprising roughly 10 and 30 per cent, respectively, of the body weight. The excretion of liberated iodide will accordingly be modified by its transport into a larger volume of distribution and also by its rate constant of renal excretion. The excretion of noniodide degradation products will be similarly modified. These considerations led to the introduction into the model of a compartment (Compartment 4) to represent degradation products not yet excreted.

\section{The model and its solution}

The simplest general model that is compatible with the various considerations outlined above is shown in Figure 3. The meaning of the compartment numbers is given in and below the figure. The model consists of six compartments, three of which (1,2 and 3) are exchanging compartments and three of which $(4,5$ and 6$)$ are nonexchanging compartments. The principal problem presented by this model is the determination of the configuration of the exchanging three-compartment system. Compartments 4, 5 and 6 , the nonexchanging or ancillary compartments, must be considered, however, if solution of the configuration of the exchanging system is to be realized.

Most complete measurements of the system were made in the case of R. B., Experiment III. Tagged albumin which could reasonably be expected to be metabolically homogeneous because of previous tenure in a donor patient was introduced into plasma (Compartment 1 ) and repeated measurements of plasma specific activity and cumulative urinary excretion of label (Compartment 5) were made (Figure 1).

$\lambda_{54}$ was assumed to be known on the basis of considerations to be discussed later. From knowledge of $q_{5}(t), q_{4}(t)$ can be calculated from the relation:

$$
\frac{\mathrm{dq}_{5}(\mathrm{t})}{\mathrm{dt}}=\lambda_{54} \mathrm{q}_{4}(\mathrm{t})
$$

and

$$
\text { 6) } \quad q_{4}(t)=\frac{1}{\lambda_{54}} \frac{\mathrm{dq}_{5}(\mathrm{t})}{\mathrm{dt}} \text {. }
$$

$\frac{d q_{5}(t)}{d t}$ can be obtained by either graphical or analytic differentiation of $q_{5}(t)$.

No measurements of the postulated Compartment 6 were made. By definition and from theoretical considerations (1) it follows that the asymptotic value of $q_{6}(t)$ must equal $1-A_{65}$, the asymptotic value of $\mathrm{q}_{5}(\mathrm{t})$, and that $\mathrm{q}_{6}(\mathrm{t})$ must have the same exponential slopes $\left(\alpha_{1}, \alpha_{2}\right.$ and $\alpha_{3}$ ) as the plasma curve. From the available data, however, solution of the individual turnover rates $\lambda_{61}, \lambda_{62}$ and $\lambda_{63}$ is not possible. To pursue further the solution of the exchanging iodoalbumin system it is helpful to get an expression for the total radioactivity in Compart- 
ments 2 and 3 as a function of time. This requires an expression for the radioactivity in Compartment 6 as a function of time. For mathematical simplicity it was assumed that the activity in Compartment 6 is proportional to the sum of the activity in Compartments 4 and 5 so that

and

$$
\mathrm{q}_{6}(\mathrm{t})=\mathrm{K}\left[\mathrm{q}_{4}(\mathrm{t})+\mathrm{q}_{5}(\mathrm{t})\right]
$$

where

$$
\mathrm{q}_{5}(\infty)+\mathrm{Kq}_{5}(\infty)=1 \text {, }
$$

$$
\mathrm{K}=\frac{1-\mathrm{q}_{5}(\infty)}{\mathrm{q}_{5}(\infty)}=\frac{1-\mathrm{A}_{55}}{\mathrm{~A}_{55}} .
$$

Although this assumption may result in completely erroneous values for $\lambda_{61}, \lambda_{62}$ and $\lambda_{63}$, its effect on the turnover rates of the exchanging system is relatively small because $q_{6}(t)$ is small compared to $\mathrm{q}_{5}(\mathrm{t})$.

From the data and the above assumptions regarding $\lambda_{54}$ and Compartment 6 , the total radioactivity in Compartments 2 and 3 can be calculated from the relation

$7 a)$

$$
\begin{aligned}
\mathrm{q}_{2}(\mathrm{t}) & +\mathrm{q}_{3}(\mathrm{t}) \\
& =1-\left[\mathrm{q}_{1}(\mathrm{t})+\mathrm{q}_{4}(\mathrm{t})+\mathrm{q}_{5}(\mathrm{t})+\mathrm{q}_{6}(\mathrm{t})\right] \\
& =1-\left[\mathrm{q}_{1}(\mathrm{t})+\frac{\mathrm{q}_{4}(\mathrm{t})+\mathrm{q}_{5}(\mathrm{t})}{\mathrm{A}_{55}}\right] .
\end{aligned}
$$

From Equations $2(b-c)$ it follows that Equation $7 a$ ) may be written:

b) $\mathrm{q}_{2}(\mathrm{t})+\mathrm{q}_{3}(\mathrm{t})$

$$
\begin{aligned}
=\left(A_{21}+A_{31}\right)_{e}^{-\alpha_{1} t} & +\left(A_{22}+A_{32}\right)_{e}^{-\alpha_{2} t} \\
& +\left(A_{23}+A_{33}\right)_{e}^{-\alpha_{3} t},
\end{aligned}
$$

where

$$
\sum_{\mathrm{i}}\left(\mathrm{A}_{2 \mathrm{i}}+\mathrm{A}_{3 \mathrm{i}}\right)=0 .
$$

The problem of the exchanging iodoalbumin system reduces to the general solution of a threecompartmental system when data on the compartment into which label is initially injected and the sum of the two other compartments is available. The considerations and solution that follow are based on the steady state analysis in Reference (1) and no effort will be made here to justify it.

The general three-compartment system (shown schematically in Figure 4 ) is characterized by nine independent fractional turnover rates, three for

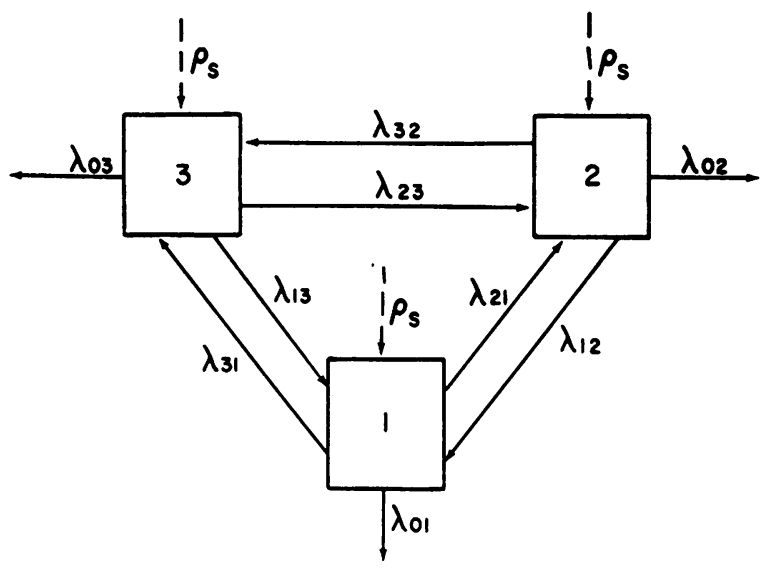

Fig. 4. The General Three-Compartment System Providing for Two Way Exchange of Any One ComPARTMENT WITH EACH OF THE OTHER TWO AND FOR ENTRY of New Carrier into, and Excretion of Carrier from, EACH OF THE COMPaRTMENTS

Such a system is characterized by the nine independent fractional turnover rates shown beside each of the appropriately directed arrows. $\lambda_{j j}$ is defined as the sum of the fractional turnover rates of compartment $j$; for example, $\lambda_{22} \equiv \lambda_{02}+\lambda_{12}+\lambda_{32}$.

each compartment. Radioactivity-time measurements of any one compartment contain five independent constants: three $\alpha$ 's and two A's. Only two of the three A's obtained from measurements of a sampled compartment are independent since the sum of the A's is fixed for each compartment (see Equation 2). Two additional independent constants (two A's) are contained in the measurements of each additional compartment. Nine measured independent constants (six A's and three $\alpha$ 's) corresponding to the nine independent fractional turnover rates ( $\lambda$ 's) are required for a complete (unique) solution of the system. The data of R. B., Experiment III, contain seven independent constants : five from $\mathrm{q}_{1}(\mathrm{t})$ (two A's and three $\alpha$ 's) and two from $\mathrm{q}_{2}(\mathrm{t})+\mathrm{q}_{3}(\mathrm{t})$ (two A's). This means that $(9-7=) 2$ independent variables are left undetermined. Consequently the data do not permit a unique solution of the system. ${ }^{3}$ This means that there exist

${ }^{3}$ Subsequently we shall speak of a system to mean a given number of compartments without regard for the values of their turnover rates or the manner of their interconnection. A model will mean a system with a specified set of turnover rates that is mathematically consistent with the experimentally measured quantities. A physically realizable model will mean one in which all the turnover rates are positive or zero. 
not one but an infinity of compatible models each of which is mathematically consistent with the experimental data.

Even though the number of models consistent with the data is infinite, the range of variation of each turnover rate for all possible compatible models may be quite restricted. It will now be shown how to obtain the range of variation of the turnover rates for all models and their values for a chosen model.

The procedures outlined follow from the treatment presented in Reference (1); they are, however, sufficiently complete so that they may be used without Reference (1). The original treatment starts with the conventional first order differential equations that describe the kinetics of isotopes. These are then transformed-using Laplace transform methods and matrix algebraso that the solutions of turnover rates are expressible in simple algebraic equations and in terms of slopes and intercepts of the data expressed as a sum of exponential terms.

In the conventional solutions of model turnover rates using differential equations it is necessary that the data be complete to determine the model uniquely. When the data are incomplete some simplified model has to be assumed and then tested to see if it is compatible with the data. In special cases, certain turnover rates may be solved for directly from the data without assuming any model. There are no criteria, however, that can be applied to determine which models are or are not compatible with the data, or which turnover rates can or cannot be solved for directly. Furthermore, if one cannot think of a special model or solve for some turnover rates, much of the information contained in the data cannot be used.

The solution presented here is different from the above in that no assumptions as to any model have to be made. A general solution is obtained that represents the solution of all models. From the general solution, one can readily deduce which models are or are not possible without necessarily solving for the turnover rates of the models. Furthermore, the general solution permits the easy calculation of the turnover rates of any desired model and the determination of the range or degree of variation of any one turnover rate for all models.

There are various ways to obtain an expression for the general solution of the system. The method presented here was chosen because of its relative simplicity. It is broken down into two steps. The first step is to obtain a solution for the turnover rates of one model consistent with the data. The second step is to express the turnover rates of the general solution in terms of the solved model rates and two variables ( $x$ and $y$ ) that express the incompleteness of the data.

As already pointed out, the data are insufficient to determine a model uniquely. Hence, to solve for some compatible model, certain assumptions must be made. Here, again, there is a difference between the conventional differential equation methods and this method. In the former, assumptions are made about the modeli.e., certain turnover rates are set to zero. Then one solves for the assumed model turnover rates and checks to see if the solution is compatible. In the method presented here, the assumptions are made about the missing data-i.e., arbitrary values are assigned to them with the only restriction being that the initial conditions be satisfied. This may result in peculiar physical models in that some turnover rates may be negative. This, however, is of no significance in this method because mathematically, such a model is consistent with the data. The general solution merely uses the values of the solved model for an expression that includes all mathematically consistent models-those that are physically realizable and nonrealizable.

The assumptions made here about the missing data to yield the solution of $a$ model are:

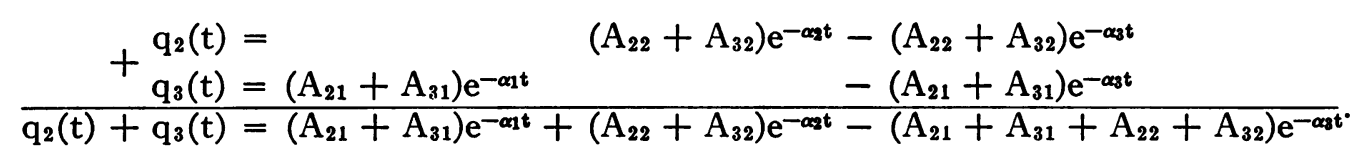

The expression for $\mathrm{q}_{2}(\mathrm{t})+\mathrm{q}_{3}(\mathrm{t})$ is the same as Equation $7 b$.

The above assumptions are arbitrary. Several other ways to obtain the solution of a model are discussed in Reference (1). 
Using these assumptions, the solution of the model turnover rates (this follows from any method of solution) are:

$8 a)$

b)

$$
\lambda_{11}{ }^{\prime}=A_{11} \alpha_{1}+A_{12} \alpha_{2}+A_{13} \alpha_{3}
$$

$\lambda_{12}^{\prime}=\frac{A_{12}}{\left(A_{22}+A_{32}\right)}\left[A_{11}\left(\alpha_{1}-\alpha_{2}\right)-A_{13}\left(\alpha_{2}-\alpha_{3}\right)\right]$

c)

$\lambda_{13}{ }^{\prime}=-\frac{\mathrm{A}_{11}}{\left(\mathrm{~A}_{21}+\mathrm{A}_{31}\right)}\left[\mathrm{A}_{12}\left(\alpha_{1}-\alpha_{2}\right)+\mathrm{A}_{13}\left(\alpha_{1}-\alpha_{3}\right)\right]$

d)

$\lambda_{21}{ }^{\prime}=-\left(\mathrm{A}_{22}+\mathrm{A}_{32}\right)\left(\alpha_{2}-\alpha_{3}\right)$

e)

f) $\quad \lambda_{23}{ }^{\prime}=\frac{\mathrm{A}_{11}\left(\mathrm{~A}_{22}+\mathrm{A}_{32}\right)}{\left(\mathrm{A}_{21}+\mathrm{A}_{31}\right)}\left(\alpha_{2}-\alpha_{3}\right)$

g) $\quad \lambda_{31}{ }^{\prime}=-\left(\mathrm{A}_{21}+\mathrm{A}_{31}\right)\left(\alpha_{1}-\alpha_{3}\right)$

h) $\quad \lambda_{32}^{\prime}=\frac{\left(\mathrm{A}_{21}+\mathrm{A}_{31}\right)\left(\mathrm{A}_{12}\right)}{\left(\mathrm{A}_{22}+\mathrm{A}_{32}\right)}\left(\alpha_{1}-\alpha_{3}\right)$

i) $\quad \lambda_{33}{ }^{\prime}=\left(\mathrm{A}_{12}+\mathrm{A}_{13}\right) \alpha_{1}+\mathrm{A}_{11} \alpha_{3}$

j) $\quad \lambda_{01}{ }^{\prime}=\left(A_{11}+A_{21}+A_{31}\right) \alpha_{1}+\left(A_{12}+A_{22}+A_{32}\right) \alpha_{2}+\left(A_{13}+A_{23}+A_{33}\right) \alpha_{3}$.

The values of the A's and $\alpha$ 's of these equations are the values of the A's and $\alpha$ 's of Equations $2 a$ and $7 b$ that fit the experimental data.

The general solution can be obtained in terms of the values of the specific solution and the two variables ( $x$ and $y$ ) that represent the "missing" data. The equations were derived using a matrix mapping technique (1) whereby the given data are kept fixed for all the variations in the unknown data. ${ }^{4}$ The general solution is:

$$
\begin{array}{ll}
\text { 9a) } & \lambda_{11}=\lambda_{11}{ }^{\prime} \\
\text { b) } & \lambda_{12}=\frac{\lambda_{12}{ }^{\prime}(1-\mathrm{y})-\lambda_{13}{ }^{\prime}(1-\mathrm{x})}{\mathrm{x}-\mathrm{y}} \\
\text { c) } & \lambda_{13}=\frac{-\lambda_{12}{ }^{\prime} \mathrm{y}+\lambda_{13}{ }^{\prime} \mathrm{x}}{\mathrm{x}-\mathrm{y}} \\
\text { d) } & \lambda_{01}=\lambda_{01}{ }^{\prime} \\
\text { e) } & \lambda_{02}=\frac{\lambda_{02}{ }^{\prime}(1-\mathrm{y})-\lambda_{03}{ }^{\prime}(1-\mathrm{x})}{\mathrm{x}-\mathrm{y}} \\
\text { f) } & \lambda_{03}=\frac{-\lambda_{02} \mathrm{y}+\lambda_{03}{ }^{\prime} \mathrm{x}}{\mathrm{x}-\mathrm{y}} \\
\text { g) } & \lambda_{21}=\lambda_{21} \mathrm{x}^{\prime}+\lambda_{31}{ }^{\prime} \mathrm{y} \\
\text { h) } & \lambda_{22}=\frac{\left(\lambda_{22}{ }^{\prime} \mathrm{x}-\lambda_{32}{ }^{\prime} \mathrm{y}\right)(1-\mathrm{y})+\left(\lambda_{23}{ }^{\prime} \mathrm{x}-\lambda_{33}{ }^{\prime} \mathrm{y}\right)(1-\mathrm{x})}{\mathrm{x}-\mathrm{y}} \\
\text { i) } & \lambda_{23}=\frac{\left(\lambda_{22}{ }^{\prime} \mathrm{x}-\lambda_{32}{ }^{\prime} \mathrm{y}\right) \mathrm{y}+\left(\lambda_{23}{ }^{\prime} \mathrm{x}-\lambda_{33}{ }^{\prime} \mathrm{y}\right) \mathrm{x}}{\mathrm{x}-\mathrm{y}}
\end{array}
$$

In matrix notation the general solution of the $|\lambda|$ matrix may be expressed in terms of any specific solution $\left|\lambda^{\prime}\right|$ matrix as

$$
|\lambda|=|P|\left|\lambda^{\prime}\right|\left|P^{-1}\right|,
$$

where the matrix $|P|$ represents a mapping that preserves the given data $q_{1}(t)$ and $q_{2}(t)+q_{3}(t)$ and is of the form:

$$
|P|=\left|\begin{array}{ccc}
1 & 0 & 0 \\
0 & x & y \\
0 & 1-x & 1-y
\end{array}\right| .
$$




$$
\begin{array}{ll}
\text { j) } & \lambda_{31}=\lambda_{21}{ }^{\prime}(1-\mathrm{x})+\lambda_{31}{ }^{\prime}(1-\mathrm{y}) \\
\text { k) } & \lambda_{32}=\frac{\left[-\lambda_{22}{ }^{\prime}(1-\mathrm{x})+\lambda_{32}{ }^{\prime}(1-\mathrm{y})\right](1-\mathrm{y})-\left[\lambda_{23}{ }^{\prime}(1-\mathrm{x})-\lambda_{33}{ }^{\prime}(1-\mathrm{y})\right](1-\mathrm{x})}{\mathrm{x}-\mathrm{y}} \\
\text { l) } & \lambda_{33}=\frac{-\left[\lambda_{22}{ }^{\prime}(1-\mathrm{x})-\lambda_{32}{ }^{\prime}(1-\mathrm{y})\right] \mathrm{y}+\left[-\lambda_{23}{ }^{\prime}(1-\mathrm{x})+\lambda_{33}{ }^{\prime}(1-\mathrm{y})\right] \mathrm{x}}{\mathrm{x}-\mathrm{y}} .
\end{array}
$$

The primes refer to any compatible model that is already solved. Such a model will be called a "generating model" when it is used to generate all other mathematically compatible models from it, as indicated by Equations $9(a-l)$.

The substitution of any value of $\mathrm{x}$ and $\mathrm{y}$ into Equations $9(a-l)$ results in the correct solution of some compatible model. On the other hand, the arbitrary assignment of some $\lambda_{i j}$ to zero results in a constraint on the x's and y's. For each $\lambda_{i j}$ a different constraint may result. Since there are only two variables and nine independent turnover rates, at most two turnover rates can in general be set equal to zero simultaneously. Thus, for example, a parallel model requires that $\lambda_{23}=\lambda_{32}=0$. This results in two simultaneous equations in $x$ and $y$, the solution of which yields $a$ value of $x$ and a value of $y$. Substitution of these values into Equations $9(a-l)$ gives the values of the turnover rates for the parallel model.

The analysis presented is adequate when the data is in the required form. Certain difficulties that arise in practice will now be discussed with regard to the data of R. B., Experiment III.

In Experiment III, two corrections of the data as obtained involving Compartment 4 required consideration. 1) The labeled plasma removed from M. H. and injected into R. B. was not dialyzed prior to injection to remove degradation products of iodoalbumin not yet excreted by the donor. A correction of the urinary excretion curve of R. B. for injected label not in the form of iodoalbumin was necessary. 2) The plasma curve was obtained from counts on unfractionated plasma which contains some Compartment 4 activity. It is apparent that suitable corrections require that Compartment 4 activity as a function of time be known for the donor and recipient.

From theoretical considerations it can be shown 1) that, for the system under consideration (see Figure 5), the cumulative urinary excretion curve is composed of four exponential components and a constant:

$$
\begin{aligned}
& \text { 10) } q_{5}(t)=A_{51 e^{-\alpha_{1} t}}+A_{52 e^{-\alpha_{2} t}}+A_{53 e^{-\alpha_{3} t}} \\
& +A_{54 e^{-\lambda 64 t}}+A_{55}
\end{aligned}
$$

where $\alpha_{1}, \alpha_{2}$ and $\alpha_{3}$ are identical to the $\alpha$ 's of the plasma curve and $\lambda_{54}$ is the fractional turnover rate of Compartment 4. Theoretically, then. $\lambda_{54}$ can be obtained directly by graphic analysis of $q_{5}(t)$. $\frac{d q_{5}(t)}{d t}$ can be obtained by graphic or analytical differentiation of $\mathrm{q}_{5}(\mathrm{t})$, and $\mathrm{q}_{4}(\mathrm{t})$ computed from Equation 6.

From a practical standpoint, however, even with frequent early urine collections, resolution of the excretion curve into all of its components

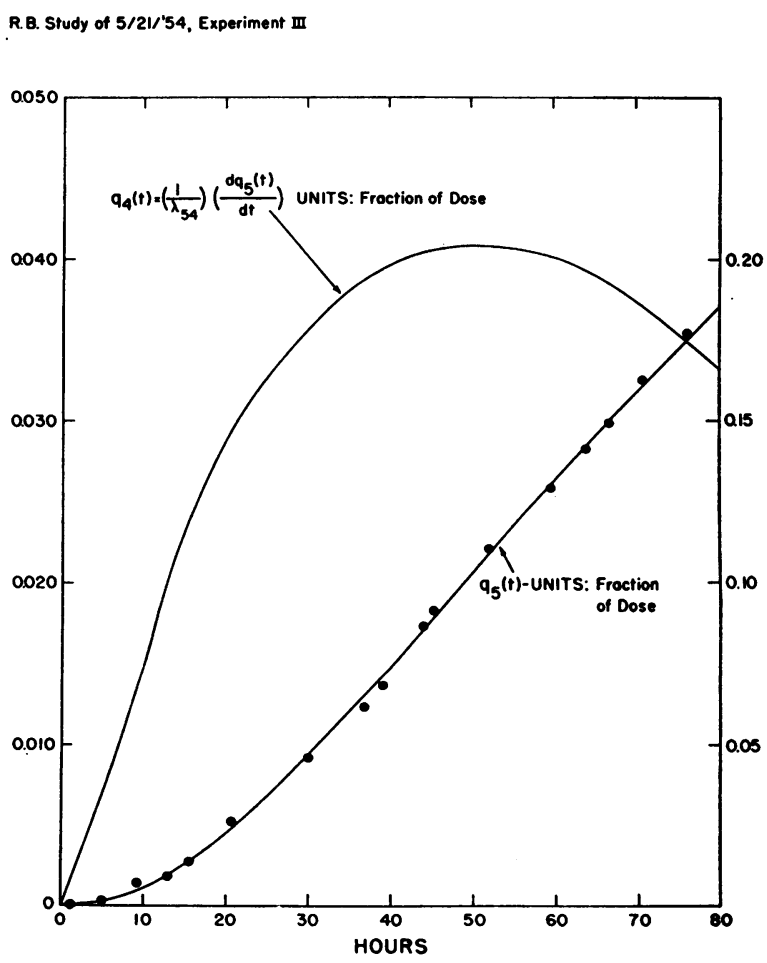

Fig. 5. Graphical Differentiation of $\mathrm{q}_{5}(\mathrm{t})$

The left ordinate of the figure refers to $q_{4}(t)$; the right ordinate to $q_{5}(t)$. 
within the restrictions imposed by the system was difficult and the reliability of the components other than the $\alpha_{3}$ component and $\mathrm{A}_{55}$ was questionable. The values of $\alpha_{1}, \alpha_{2}$ and $\alpha_{3}$ were considered to have been reliably determined from the plasma data and could be used as guides in resolving the components of the excretion curve. However, since the $\lambda_{54}$ component does not appear in the plasma data (see Equation 2a), it was desirable to have an independent measure of this constant.

In the fractionation study of urinary excretion forms of label performed on G. S., the label released by degradation of iodoalbumin appeared in the urine as 0.875 iodide and 0.125 diiodotyrosine (mean of two 24 hour collections). Studies by Albert and Keating (10) indicate that the rate constant of renal excretion of diiodotyrosine $\left(\mathrm{K}_{\mathrm{de}}\right)$ is approximately 0.169 that of iodide $\left(\mathrm{K}_{\mathrm{ie}}\right)$. Since $\lambda_{54}$ is the mean rate constant of excretion of all forms of label from degraded iodoalbumin, it was approximated as a weighted average by the relationship:

$$
\text { 11) } \begin{array}{r}
\lambda_{54} \cong 0.875 \mathrm{~K}_{\mathrm{ie}}+0.125(0.169) \mathrm{K}_{\mathrm{ie}} \\
=0.896 \mathrm{~K}_{\mathrm{ie}} .
\end{array}
$$

Following completion of the iodoalbumin experiment $\mathrm{K}_{\mathrm{ie}}$ was determined for both the donor and recipient by independent $\mathrm{NaI}^{131}$ studies according to the method outlined by Keating, Power, Berkson and Haines (13) and $\lambda_{54}$ for each was computed from the relationship in Equation 11 .

To estimate the volume of distribution of Compartment 4 , iodide space was accepted as 0.34 of the body weight (12) and diiodotyrosine space as 0.53 of the body weight (10). This gave, as a weighted mean, a volume of distribution for Compartment 4 of 0.364 of the body weight.

In the case of the donor $\mathrm{q}_{4}(\mathrm{t})$ was computed from Equation 6 for the time of withdrawal of labeled plasma. When the difference in distribution volumes of Compartments 4 and 1 was taken into account, it was calculated that approximately 0.018 of the label given to the recipient had not been in the form of iodoalbumin. The urinary excretion curve of the recipient was accordingly corrected for this quantity of label excreted at the rate of the value of $\lambda_{54}$. Similar calculations for 11 tracer studies in Experiments I, II and III indicated a mean maximum error of overestimation of plasma specific activity of 1.5 per cent from failure to fractionate plasma samples. In these studies this correction was disregarded. This computed noniodoalbumin label in plasma samples is similar to the values obtained by trichloroacetic acid precipitation (7).

The plasma specific activity, cumulative retention and cumulative urinary excretion curves for R. B., Experiment III, are shown in Figure 1. The exponential constants $\left(\alpha_{i}\right)$ and the coefficients $\left(\mathrm{X}_{1 \mathrm{i}}\right)$ of the plasma curve were determined by standard methods of graphic analysis (3). The coefficients $X_{1 i}$ were normalized by Equation 3. $\lambda_{54}$ was computed from Equation 11 . $\frac{d q_{5}(t)}{d t}$ was obtained by graphical differentiation of $\mathrm{q}_{5}(\mathrm{t})$ and $\mathrm{q}_{4}(\mathrm{t})$ was computed from Equation 6 (see Figure 5). Finally, $\mathrm{q}_{2}(\mathrm{t})+\mathrm{q}_{3}(\mathrm{t})$ was computed from Equation $7 a$. The values of the various constants were:

$$
\begin{array}{ll}
\mathrm{A}_{11}: 0.146 & \alpha_{1}: 0.180 \\
\mathrm{~A}_{12}: 0.326 & \alpha_{2}: 0.0301 \\
\mathrm{~A}_{13}: 0.528 & \alpha_{3}: 0.00339 \\
& \lambda_{54}: 0.0739
\end{array}
$$

It was now desirable to resolve the curve of $\mathrm{q}_{2}(\mathrm{t})+\mathrm{q}_{3}(\mathrm{t})$ into its exponential components so as to obtain an expression for $\mathrm{q}_{2}(\mathrm{t})+\mathrm{q}_{3}(\mathrm{t})$ having the form of Equation $7 b$. The plot of $\mathrm{q}_{2}(\mathrm{t})+\mathrm{q}_{3}(\mathrm{t})$ obtained from the experimental data is shown in Figure 6 as Curve A. In fitting exponential components to this curve the following conditions were preserved:

a) $\left(\mathrm{A}_{21}+\mathrm{A}_{31}\right)+\left(\mathrm{A}_{22}+\mathrm{A}_{32}\right)$

b) that the exponential constants be the same
as those of the plasma curve (see Equation $2 a-c$ ).

Rather than reiterate the various approaches used in fitting the curve, it is perhaps more instructive to discuss Curve $B$, which represents the sum of the components finally accepted as best fitting the curve. Inspection of the figure shows that the fit of the $\alpha_{3}$ component with $\left(A_{23}+A_{33}\right)=0.431$ is exact. Acceptance of the value of $\left(A_{23}+A_{33}\right)$ fixes the sum of $\left(A_{21}+A_{31}\right)$ $+\left(\mathrm{A}_{22}+\mathrm{A}_{32}\right)$ from condition $\left.a\right)$ above. Now 
R.B., STUDY OF $5 / 21 / 54$ - EXPERIMENT III

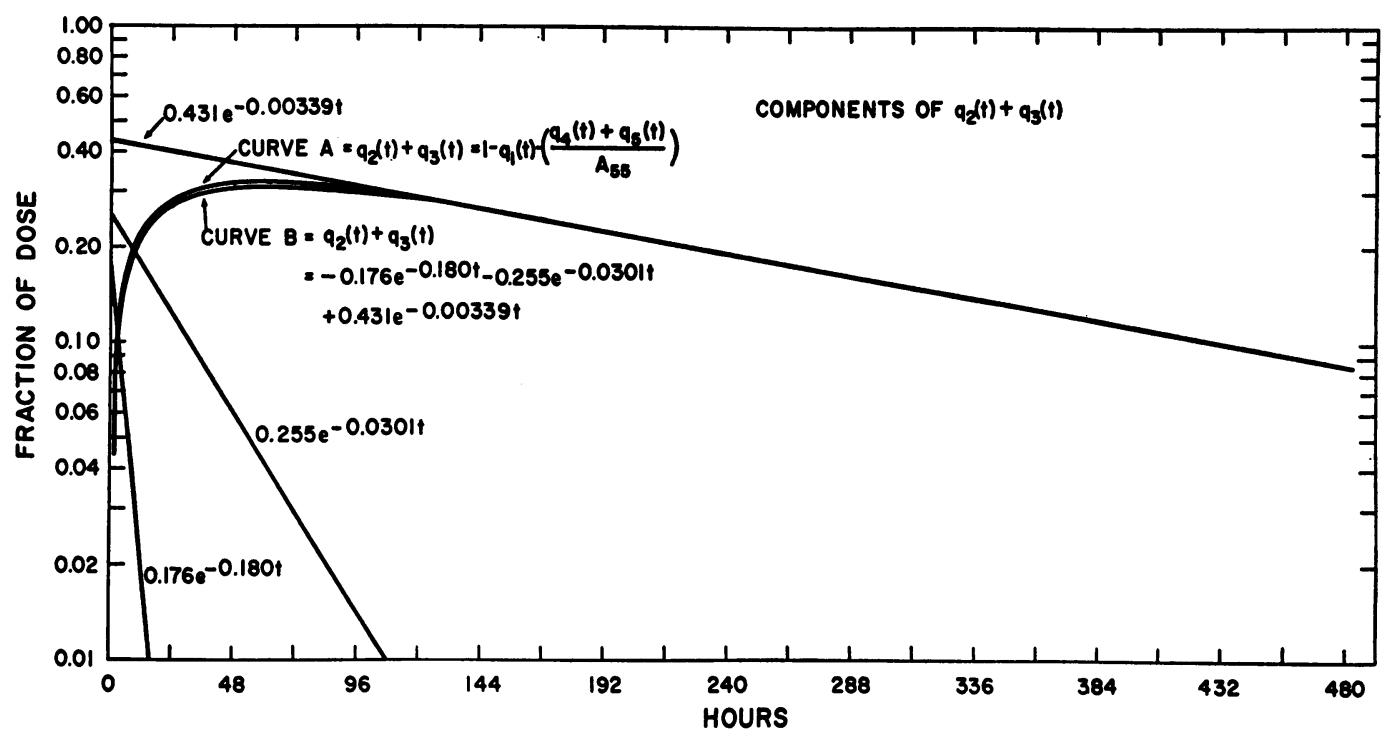

Fig. 6. Resolution of the Components of the Curve $q_{2}(t)+q_{3}(t)$

Curve $A$ is readily calculable from the experimental data. Curve $B$ represents the sum of the "best fit" components obtained by procedures described in the text.

inspection of Equations $8 j$ and $9 d$ shows that $\lambda_{01}$ is uniquely determined from the measured or readily calculable quantities $\mathrm{q}_{1}(\mathrm{t})$ and $\mathrm{q}_{2}(\mathrm{t})+$ $\mathrm{q}_{3}(\mathrm{t})$ and is therefore independent of the choice of model. It follows that $\lambda_{01}{ }^{\prime}=\lambda_{01} \geqq 0$ if a physically realizable model exists. Setting Equation $8 j \geqq 0$, and substituting known values for $\mathrm{A}_{1 \mathrm{i}}$ and $\alpha_{\mathrm{i}}$ and 0.431 for $\left(\mathrm{A}_{23}+\mathrm{A}_{33}\right)$ in condition $a)$, and solving simultaneously one obtains:

$$
\begin{aligned}
& \left(A_{22}+A_{32}\right) \leqq-0.255 \\
& \left(A_{21}+A_{31}\right) \geqq-0.176 .
\end{aligned}
$$

Curve $B$ represents the sum of the fitted components for $\lambda_{01}=0:\left(A_{21}+A_{31}\right)=-0.176$ $\left(A_{22}+A_{32}\right)=-0.255$, and $\left(A_{23}+A_{33}\right)=0.431$.

TABLE I

Solution of data of R. B., Experiment III. Fractional turnover rates for four compatible and physically

\begin{tabular}{|c|c|c|c|c|c|}
\hline & \multicolumn{5}{|c|}{ Model designation } \\
\hline & $\begin{array}{l}\lambda_{23}=\lambda_{32}=0 \\
x=-0.27916 \\
y=0.94621\end{array}$ & $\begin{array}{l}\lambda_{22}=\lambda_{21}=0 \\
x=-0.38466 \\
y=1.3038 \\
y=0\end{array}$ & $\begin{array}{l}\lambda_{31}=\lambda_{03}=0 \\
x=-0.35422 \\
y=1.2971\end{array}$ & $\begin{array}{l}\lambda_{32}=\lambda_{08}=0 \\
x=-0.25862 \\
y=0.94704\end{array}$ & $\begin{array}{c}\text { "Generating" } \\
\text { model } \\
\mathbf{x}=\mathbf{1} \\
\mathbf{y}=\mathbf{0}\end{array}$ \\
\hline$\lambda_{11}$ & $0.03788 \dagger$ & 0.03788 & 0.03788 & 0.03788 & 0.03788 \\
\hline$\lambda_{12}$ & 0.1236 & $0.09495 \dagger$ & 0.09495 & $0.1236 \dagger$ & -0.009942 \\
\hline$\lambda_{13}$ & 0.01920 & $0.01920 \dagger$ & $0.01749 \dagger$ & 0.01749 & 0.1180 \\
\hline$\lambda_{01}$ & $0 \dagger$ & 0 & 0 & 0 & 0 \\
\hline$\lambda_{02}$ & 0.03230 & $0.02359 \dagger$ & 0.02359 & $0.03230 \dagger$ & -0.008355 \\
\hline$\lambda_{03}$ & 0.0005184 & $0.0005184 \dagger$ & $0 \dagger$ & 0 & 0.03059 \\
\hline$\lambda_{21}$ & $0.02749 \dagger$ & $0.03788 \dagger$ & 0.03788 & 0.02766 & 0.006816 \\
\hline$\lambda_{22}$ & 0.1559 & 0.1559 & $0.1551 \dagger$ & $0.1559 \dagger$ & 0.02139 \\
\hline$\lambda_{28}$ & $0 \dagger$ & 0 & $0.003052 \dagger$ & 0.002228 & 0.005657 \\
\hline$\lambda_{31}$ & $0.01039 \dagger$ & $0 \dagger$ & 0 & 0.01022 & 0.03107 \\
\hline$\lambda_{32}$ & $0 \dagger$ & $0.03734 \dagger$ & 0.03652 & 0 & 0.03969 \\
\hline$\lambda_{33}$ & $0.01972 \dagger$ & 0.01973 & $0.02055 \dagger$ & 0.01972 & 0.1542 \\
\hline
\end{tabular}
realizable models and a compatible "generating" model *

* $\lambda$ values are presented to four figures to preserve the internal consistency of the model solutions and are not considered to reflect experimental precision.

† An upper or lower limiting value of the respective turnover rate for all physically realizable models. 
To avoid a negative value of $\lambda_{01},\left(\mathrm{~A}_{22}+\mathrm{A}_{32}\right)$ and $\left(A_{21}+A_{31}\right)$ may vary only as shown above. Inspection of Figure 6 shows that Curve $B$ fits the experimental Curve $A$ at the beginning and end of the curves but that the discrepancy occurs in a region where the contribution of the $\alpha_{1}$ component to the curve is negligible. From the figure it is clear that if $\left(\mathrm{A}_{22}+\mathrm{A}_{32}\right)$ and $\left(\mathrm{A}_{21}+\mathrm{A}_{31}\right)$ are allowed to take on values consistent with a positive value of $\lambda_{01}$ as indicated above, the discrepancy between Curves $A$ and $B$ will increase. It was concluded that the component values of Curve $B$ represented the best obtainable fit of the data that would avoid a negative value of $\lambda_{01}$.

These values of the coefficients of $q_{2}(t)+q_{3}(t)$ and the values previously given for $A_{1 i}, \alpha_{i}$ and $\lambda_{54}$, when substituted in Equations $8(a-j)$, yielded the solution of the generating model shown in Table I. It can be seen that this generating model has two negative fractional turnover rates. This does not matter since it is merely a mathematically compatible model. All other compatible models are expressed in terms of the variables $\mathrm{x}$ and $\mathrm{y}$ and the turnover rates of the generating model [see Equations $9(a-l)]$. The values of $\mathrm{x}$ and $\mathrm{y}$ that yield physically realizable models will be determined later.

Equations $9(a-l)$ indicate that if any turnover rate that is a function of $\mathrm{x}$ and $\mathrm{y}$ is set equal to zero, a constraint on $\mathrm{x}$ and $\mathrm{y}$ results. For ex-

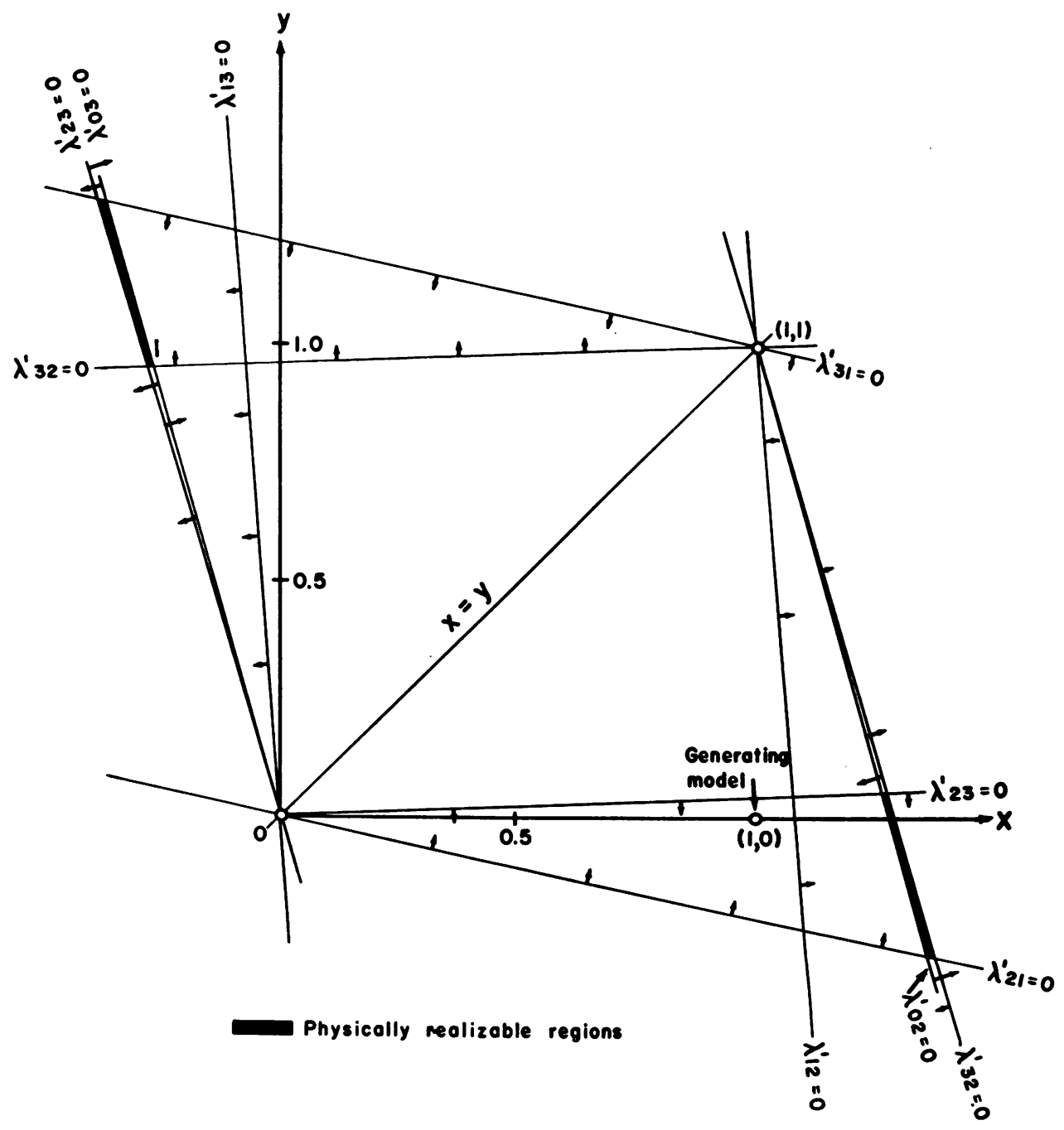

Fig. 7. A Plot of the Linear Relationships Between x and y Obtained by Setting the Respective Turnover Rates to Zero 
ample, if

$$
\begin{aligned}
\lambda_{12} & =\frac{\lambda_{12}{ }^{\prime}(1-\mathrm{y})-\lambda_{13}{ }^{\prime}(1-\mathrm{x})}{\mathrm{x}-\mathrm{y}}=0 \\
\frac{1-\mathrm{y}}{1-\mathrm{x}} & =\frac{\lambda_{13}{ }^{\prime}}{\lambda_{12}{ }^{\prime}}
\end{aligned}
$$

and if

$$
\lambda_{03}=0, \quad \frac{\mathrm{y}}{\mathrm{x}}=\frac{\lambda_{03}{ }^{\prime}}{\lambda_{12}{ }^{\prime}}, \text { etc. }
$$

Substituting the values of the $\lambda_{i j}$ 's of the generating model into each of the possible constraints of $\mathrm{x}$ and $\mathrm{y}$, one obtains the following:

$$
\begin{aligned}
& \text { for a) } \lambda_{21}=0, \quad \frac{\mathrm{y}}{\mathrm{x}}=-0.219 \\
& \text { b) } \lambda_{31}=0, \quad \frac{1-\mathrm{y}}{1-\mathrm{x}}=-0.219 \\
& \text { c) } \lambda_{01} \neq 0, \text { no constraint since } \lambda_{01} \text { is con- } \\
& \text { stant for all models } \\
& \text { d) } \lambda_{12}=0, \quad \frac{1-\mathrm{y}}{1-\mathrm{x}}=-11.9 \\
& \text { e) } \lambda_{32}=0, \frac{1-\mathrm{y}}{1-\mathrm{x}}=-3.39,0.0421 \\
& \text { f) } \lambda_{02}=0, \frac{1-\mathrm{y}}{1-\mathrm{x}}=-3.66 \\
& \text { g) } \lambda_{13}=0, \frac{\mathrm{y}}{\mathrm{x}}=-11.9 \\
& \text { h) } \lambda_{23}=0, \frac{\mathrm{y}}{\mathrm{x}}=0.0421,-3.39 \\
& \text { i) } \lambda_{03}=0, \frac{\mathrm{y}}{\mathrm{x}}=-3.66
\end{aligned}
$$

Each of these constraints results in a linear relation between $\mathrm{x}$ and $\mathrm{y}$ and is plotted as a straight line in Figure 7. It follows that if the plotted lines yield values of zero for the corresponding turnover rates, that on one side of the line the values will be positive and on the other side negative. The positive and negative sides can be determined by carefully checking each constraint. The arrows in Figure 7 indicate the direction where the positive values of the $\lambda$ 's must lie. Examination of all the arrows shows that only in the shaded regions can all the turnover rates be zero or positive. These regions therefore correspond to all the physically realiz- able models. Any value of $\mathrm{x}$ and $\mathrm{y}$ in these regions substituted in Equations $9(a-l)$ yields a mathematically compatible and physically realizable solution.

Figure 7 shows two shaded regions where physically realizable models can lie. The two regions are actually symmetric with respect to each other, in that Compartments 2 and 3 are interchanged. This results from the fact that the data give the sum of $\mathrm{q}_{2}(\mathrm{t})+\mathrm{q}_{3}(\mathrm{t})$ and the solution does not distinguish between the two. For the purposes of this discussion we shall neglect the region in the lower right side and speak of the other one only. The position of the generating model is also shown in the plot.

Figure 7 shows that only the following turnover rates (in addition to $\lambda_{01}$ ) may be zero: $\lambda_{23}, \lambda_{03}, \lambda_{31}$ and $\lambda_{32}$, and only the following combinations may be zero simultaneously:
a) $\lambda_{32}=\lambda_{23}=0$
c) $\lambda_{31}=\lambda_{03}=0$
b) $\lambda_{23}=\lambda_{31}=0$
d) $\lambda_{32}=\lambda_{03}=0$.

The values of $\mathrm{x}$ and $\mathrm{y}$ for each of these four mathematically compatible and physically realizable "simplest" models and the values of their turnover rates obtained from Equations $9(a-l)$ are shown in Table I. They are termed "simplest" because they require the minimum number of interconnections.

Complete knowledge of all the turnover rates in the system is not sufficient to determine the compartment sizes; the site of entry and the rate of synthesis of newly synthesized carrier must also be known. Assuming each of the three exchanging compartments in turn to be the sole site of entry of newly synthesized carrier into the system we can write, for entry into Compartment 1:

$$
\begin{aligned}
& \text { 12a) } \quad \mathrm{S}_{1}{ }^{\prime}=\frac{\lambda_{22}{ }^{\prime} \lambda_{33}{ }^{\prime}-\lambda_{23}{ }^{\prime} \lambda_{32}{ }^{\prime}}{\alpha_{1} \alpha_{2} \alpha_{3}} \rho_{\mathrm{s}}{ }^{\prime} \\
& \text { b) } \quad \mathrm{S}_{2}{ }^{\prime}=\frac{\lambda_{21}{ }^{\prime} \lambda_{33}{ }^{\prime}+\lambda_{31}{ }^{\prime} \lambda_{23}{ }^{\prime}}{\alpha_{1} \alpha_{2} \alpha_{3}} \rho_{\mathrm{s}}{ }^{\prime} \\
& \text { c) } \quad \mathrm{S}_{3}{ }^{\prime}=\frac{\lambda_{21}{ }^{\prime} \lambda_{32}{ }^{\prime}+\lambda_{31}{ }^{\prime} \lambda_{22}{ }^{\prime}}{\alpha_{1} \alpha_{2} \alpha_{3}} \rho_{\mathrm{s}}{ }^{\prime},
\end{aligned}
$$

where $\rho_{s}^{\prime}$ is the rate of entry of newly synthesized carrier into the system per unit time. For entry 
into Compartment 2:

$$
\mathrm{S}_{1}{ }^{\prime}=\frac{\lambda_{12}{ }^{\prime} \lambda_{33}{ }^{\prime}+\lambda_{13}{ }^{\prime} \lambda_{32}{ }^{\prime}}{\alpha_{1} \alpha_{2} \alpha_{3}} \rho_{\mathrm{s}}{ }^{\prime}
$$

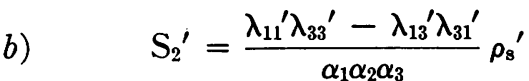$$
\text { c) } \quad \mathrm{S}_{3}{ }^{\prime}=\frac{\lambda_{11}{ }^{\prime} \lambda_{32}{ }^{\prime}+\lambda_{12}{ }^{\prime} \lambda_{31}{ }^{\prime}}{\alpha_{1} \alpha_{2} \alpha_{3}} \rho_{\mathbf{3}}{ }^{\prime} \text {. }
$$

For entry into Compartment 3 :

$$
\mathrm{S}_{1}{ }^{\prime}=\frac{\lambda_{12}{ }^{\prime} \lambda_{23}{ }^{\prime}+\lambda_{22}{ }^{\prime} \lambda_{13}{ }^{\prime}}{\alpha_{1} \alpha_{2} \alpha_{3}} \rho_{\mathrm{s}}{ }^{\prime}
$$$$
\text { b) } \quad \mathrm{S}_{2}{ }^{\prime}=\frac{\lambda_{11}{ }^{\prime} \lambda_{23}{ }^{\prime}+\lambda_{21}{ }^{\prime} \lambda_{13}{ }^{\prime}}{\alpha_{1} \alpha_{2} \alpha_{3}} \rho_{\mathrm{s}}{ }^{\prime}
$$$$
\text { c) } \quad \mathrm{S}_{3}{ }^{\prime}=\frac{\lambda_{11}{ }^{\prime} \lambda_{22}{ }^{\prime}-\lambda_{12}{ }^{\prime} \lambda_{21}{ }^{\prime}}{\alpha_{1} \alpha_{2} \alpha_{3}} \rho_{\mathrm{s}}{ }^{\prime} \text {. }
$$

Now $S_{1}$ is independent of the model chosen:

15) $\mathrm{S}_{1}=\mathrm{S}_{1}{ }^{\prime}=$ constant $=\frac{1}{\sum_{\mathrm{i}} \mathrm{X}_{1 \mathrm{i}}}$.

Accordingly for any assumed or known site of entry, $\mathrm{S}_{1}{ }^{\prime}$ may be obtained from Equation 15 and $\rho_{8}{ }^{\prime}, S_{2}{ }^{\prime}$ and $S_{3}{ }^{\prime}$ computed by appropriate substitution in Equation 12,13 or 14 . Furthermore, for entry in to Compartment 1 , the solution for all models in terms of $\mathrm{x}$ and $\mathrm{y}$ is :

16a) $\quad \mathrm{S}_{2}=\mathrm{x}_{2}{ }^{\prime}+\mathrm{y} \mathrm{S}_{3}{ }^{\prime}$

b) $\quad S_{3}=(1-x) S_{2}{ }^{\prime}+(1-y) S_{3}{ }^{\prime}$.

From Equation 16 it follows that $S_{2}+S_{3}=$ $\mathrm{S}_{2}{ }^{\prime}+\mathrm{S}_{3}{ }^{\prime}=$ constant for all models with Compartment 1 as the site of entry and $S_{1}+S_{2}+S_{3}$ $=\mathrm{S}_{1}{ }^{\prime}+\mathrm{S}_{2}{ }^{\prime}+\mathrm{S}_{3}{ }^{\prime}=$ constant for all such models. The values of $S_{j}$ and $\rho_{\mathbf{s}}$ for the four simplest models are tabulated in Table II, assuming in turn each of the exchanging compartments to be the site of entry of newly synthesized albumin into the system. The considerable dependence of $\rho_{\mathrm{s}}$ and $\mathrm{S}_{\mathrm{j}}$ on the site of entry is apparent.

The amount of carrier albumin that flows to any compartment $\mathrm{i}$ from any other compartment $\mathrm{j}$ may be computed from the relation

$$
\rho_{\mathrm{ij}}=\lambda_{\mathrm{ij}} \mathrm{S}_{\mathrm{j}} .
$$

Since all physically realizable models are expressed as a region in the $\mathrm{x}-\mathrm{y}$ plane, the range of variation of each turnover rate for all permissible models can be obtained by investigating

TABLE II

\begin{tabular}{|c|c|c|c|c|c|c|}
\hline \multirow[b]{2}{*}{$\begin{array}{l}\text { Site of carrier } \\
\text { entry }\end{array}$} & & \multicolumn{5}{|c|}{ Model designation } \\
\hline & & $\lambda_{23}=\lambda_{32}=0$ & $\lambda_{23}=\lambda_{31}=0$ & $\lambda_{31}=\lambda_{03}=0$ & $\lambda_{32}=\lambda_{03}=0$ & $\begin{array}{c}\text { "Generating" } \\
\text { model }\end{array}$ \\
\hline Compartment 1 & $\begin{array}{l}S_{1} \\
S_{2} \\
S_{3} \\
S_{2}+S_{3} \\
S_{1}+S_{2}+S_{3} \\
\rho_{3} \\
\lambda_{\mathrm{OT}} \dagger\end{array}$ & $\begin{array}{c}103.09 \\
18.19 \\
54.31 \\
72.50 \\
175.59 \\
0.6159 \\
0.003508\end{array}$ & $\begin{array}{c}103.09 \\
25.06 \\
47.44 \\
72.50 \\
175.59 \\
0.6159 \\
0.003508\end{array}$ & $\begin{array}{c}103.09 \\
26.10 \\
46.40 \\
72.50 \\
175.59 \\
0.6159 \\
0.003508\end{array}$ & $\begin{array}{c}103.09 \\
19.06 \\
53.44 \\
72.50 \\
175.59 \\
0.6159 \\
0.003508\end{array}$ & $\begin{array}{c}103.09 \\
41.14 \\
31.36 \\
72.50 \\
175.59 \\
0.6159 \\
0.003508\end{array}$ \\
\hline Compartment 2 & $\begin{array}{l}\mathrm{S}_{1} \\
\mathrm{~S}_{2} \\
\mathrm{~S}_{3} \\
\mathrm{~S}_{2}+\mathrm{S}_{3} \\
\mathrm{~S}_{1}+\mathrm{S}_{2}+\mathrm{S}_{3} \\
\rho_{\mathrm{s}} \\
\lambda_{\mathrm{OT}} \dagger\end{array}$ & $\begin{array}{c}103.09 \\
23.17 \\
54.31 \\
77.48 \\
180.57 \\
0.7769 \\
0.004303\end{array}$ & $\begin{array}{c}103.09 \\
29.75 \\
56.31 \\
86.06 \\
189.15 \\
0.7311 \\
0.003865\end{array}$ & $\begin{array}{c}103.09 \\
30.98 \\
55.08 \\
86.06 \\
189.15 \\
0.7311 \\
0.003865\end{array}$ & $\begin{array}{c}103.09 \\
24.04 \\
53.44 \\
77.48 \\
180.57 \\
0.7769 \\
0.004302\end{array}$ & $\begin{array}{c}103.09 \\
71.29 \\
39.12 \\
110.40 \\
213.49 \\
0.6014 \\
0.002817\end{array}$ \\
\hline Compartment 3 & $\begin{array}{l}\mathrm{S}_{1} \\
\mathrm{~S}_{2} \\
\mathrm{~S}_{3} \\
\mathrm{~S}_{2}+\mathrm{S}_{3} \\
\mathrm{~S}_{1}+\mathrm{S}_{2}+\mathrm{S}_{3} \\
\rho_{\mathrm{s}} \\
\lambda_{\mathrm{OT}} \dagger\end{array}$ & $\begin{array}{c}103.09 \\
18.18 \\
86.39 \\
104.57 \\
207.66 \\
0.6328 \\
0.003047\end{array}$ & $\begin{array}{c}103.09 \\
25.05 \\
79.52 \\
104.57 \\
207.66 \\
0.6328 \\
0.003047\end{array}$ & $\begin{array}{c}103.09 \\
26.73 \\
78.19 \\
104.92 \\
208.01 \\
0.6307 \\
0.003032\end{array}$ & $\begin{array}{c}103.09 \\
19.51 \\
85.40 \\
104.91 \\
208.00 \\
0.6307 \\
0.003032\end{array}$ & $\begin{array}{c}103.09 \text { 政 } \\
42.55 \\
36.69=1 \\
79.24 \\
182.33 \\
0.7675 \\
0.004209\end{array}$ \\
\hline
\end{tabular}

Solution of data of R. B., Experiment III. The effect of the site of entry of newly synthesized carrier into the exchanging system on $S_{j}, \rho_{s}$, and $\lambda_{0 T}$ for four compatible, physically realizable models and a compatible "generating" model *

* Solution values are presented to an arbitrary four or five figures to preserve the internal consistency of the model solutions and are not considered to reflect experimental precision.

† Compare values to $\alpha_{3}=0.00339$. 
their variation over the entire region. The maximum and minimum values of each turnover rate for all physically realizable models are indicated in Table I.

The specific activity of any compartment may be defined as the ratio of labeled to carrier albumin and is a function of time, the compartment sizes and the turnover rates. It is frequently desirable to express the ratio between the specific activities of two or more compartments for large values of time. This can be expressed as

$$
\frac{x_{i}}{x_{j}}=\frac{A_{i n} S_{j}}{A_{j n} S_{i}} \text { for } t \gg 0 \text {. }
$$

In general $S_{j} / S_{i}$ is dependent on the model and the rate of synthesis and site of entry of carrier. If it is assumed that newly synthesized carrier enters Compartment 1 only, then

18a) $\begin{aligned} \frac{\mathrm{x}_{2}}{\mathrm{x}_{1}}=\left[\frac{\lambda_{22} \lambda_{33}-\lambda_{23} \lambda_{32}}{\left(\lambda_{22}-\alpha_{3}\right)\left(\lambda_{33}-\alpha_{3}\right)-\lambda_{23} \lambda_{32}}\right] \\ \times\left[\frac{\lambda_{21}\left(\lambda_{33}-\alpha_{3}\right)+\lambda_{31} \lambda_{23}}{\lambda_{21} \lambda_{33}+\lambda_{31} \lambda_{23}}\right] \text { for } t \gg 0 .\end{aligned}$

Similarly,

$$
\begin{aligned}
\frac{\mathrm{x}_{3}}{\mathrm{x}_{1}}=\left[\frac{\lambda_{22} \lambda_{33}-\lambda_{23} \lambda_{32}}{\left(\lambda_{22}-\alpha_{3}\right)\left(\lambda_{33}-\alpha_{j}\right)-\lambda_{23} \lambda_{32}}\right] \\
\times\left[\frac{\lambda_{21} \lambda_{32}+\lambda_{31}\left(\lambda_{22}-\alpha_{3}\right)}{\lambda_{21} \lambda_{32}+\lambda_{31} \lambda_{22}}\right] \\
\text { for } t \gg 0 .
\end{aligned}
$$

It can be shown that the first bracketted term on the right hand side of these equations is independent of the model chosen. The second term, however, does depend on the model chosen. For the special case of a parallel model $\left(\lambda_{23}=\lambda_{32}=0\right)$ these equations reduce to

$$
\begin{aligned}
& \frac{\mathrm{x}_{2}}{\mathrm{x}_{1}}=\frac{\lambda_{22}}{\lambda_{22}-\alpha_{3}} \text { for } \mathrm{t} \gg 0 \\
& \frac{\mathrm{x}_{3}}{\mathrm{x}_{1}}=\frac{\lambda_{33}}{\lambda_{33}-\alpha_{3}} \text { for } \mathrm{t} \gg 0 .
\end{aligned}
$$

In this connection, the data of C. C. shown in Figure 5 of Reference (2) are of considerable interest. Shortly after the beginning of treatment with $\mathrm{L}$-triiodothyronine there is an apparent rise in plasma specific activity which would require entry into the plasma of labeled material from a compartment having a specific activity greater than that of plasma.
From Figure 7 it is apparent that the parallel model $\left(\lambda_{23}=\lambda_{32}=0\right)$ is a physically realizable model but that the series model is excluded (the intersection of the lines $\lambda_{31}{ }^{\prime}=0$ and $\lambda_{13}{ }^{\prime}=0$ is outside the shaded area). From Tables I and II it can be seen from the solutions of the four simplest limiting models that in general the exchange of albumin (grams per hour) across the boundary between Compartments 2 and 3 is smaller than that across the boundaries between Compartments 1 and 3 or 1 and 2. These facts and certain physiological considerations lead us to favor the parallel model as the one most closely resembling the biological system. Further application of the parallel model solution will be considered elsewhere (2). Once a model has been chosen, it is of considerable interest to construct curves of radioactivity and specific activity as a function of time for each of the compartments as this serves to clarify certain implications of the solution. For the parallel model with the ancillary Compartments 4, 5 and 6 having the configuration shown in Figure 3, the theoretical equations for radioactivity as a function of time in Compartments 1, 2, 3 and 5 are given above (Equations 2 and 10 ). The corresponding equations for Compartments 4 and 6 are:

$$
\begin{aligned}
\mathrm{q}_{4}(\mathrm{t})=\mathrm{A}_{41 \mathrm{e}^{-\alpha_{1} t}}+\mathrm{A}_{42 \mathrm{e}^{-\alpha_{2} t}} & +\mathrm{A}_{43 \mathrm{e}^{-\alpha_{3} t}}+\mathrm{A}_{44 \mathrm{e}^{-\lambda_{64} t}}
\end{aligned}
$$

where

$$
\sum_{i} A_{4 i}=0
$$

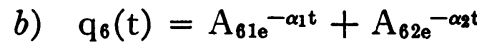

where

$$
+A_{63 e^{-\alpha 3 t}}+A_{64}
$$

$$
\sum_{i} A_{6 i}=0 .
$$

The following additional relationships, together with the solution given in Table I, may be used to compute the coefficients $A_{j i}$ for all nonsampled compartments and for experimental compartment curves not completely resolved into exponential components.

$$
\begin{aligned}
& \text { 20a) } \quad \mathrm{A}_{2 \mathrm{i}}=\frac{\lambda_{21}}{\lambda_{22}-\alpha_{\mathrm{i}}} \mathrm{A}_{1 \mathrm{i}} \\
& \text { b) } \mathrm{A}_{3 \mathrm{i}}=\frac{\lambda_{31}}{\lambda_{33}-\alpha_{\mathrm{i}}} \mathrm{A}_{1 \mathrm{i}} \\
& \text { c) } \quad \lambda_{6 \mathrm{j}}=\lambda_{0 \mathrm{j}}\left(1-\mathrm{A}_{65}\right)=\lambda_{0 \mathrm{j}} \mathrm{A}_{64} \\
& \text { d) } \quad \lambda_{4 \mathrm{j}}=\lambda_{0 \mathrm{j}} \mathrm{A}_{65}
\end{aligned}
$$


e) $\quad \mathrm{A}_{4 \mathrm{i}}=\frac{\mathrm{A}_{1 \mathrm{i}} \lambda_{41}+\mathrm{A}_{2 \mathrm{i}} \lambda_{42}+\mathrm{A}_{3 \mathrm{i}} \lambda_{43}}{\lambda_{54}-\alpha_{\mathrm{i}}}$

$$
\mathrm{A}_{44}=-\sum_{\mathrm{i}=1}^{3} \mathrm{~A}_{4 \mathrm{i}}
$$

f) $\quad \mathrm{A}_{5 \mathrm{i}}=-\frac{\mathrm{A}_{4 \mathrm{i}} \lambda_{54}}{\alpha_{\mathrm{i}}} ; \mathrm{A}_{54}=-\mathrm{A}_{44}$

g) $A_{6 i}=\left(A_{4 i}+A_{6 i}\right) \frac{A_{64}}{A_{65}}$.
By substitution of the coefficients $A_{j i}$ in Equations 2, 10 and 19 , radioactivity-time curves, and, with appropriate normalization, specific activitytime curves for all compartments may be constructed. These plots for the tracer study under discussion are shown in Figure 8. Compartment 1 is assumed to be the site of entry of newly synthesized carrier. The inequality of specific activities in the exchanging compartments for

R. B.; Study of $5 / 21 / 254$, Experiment III

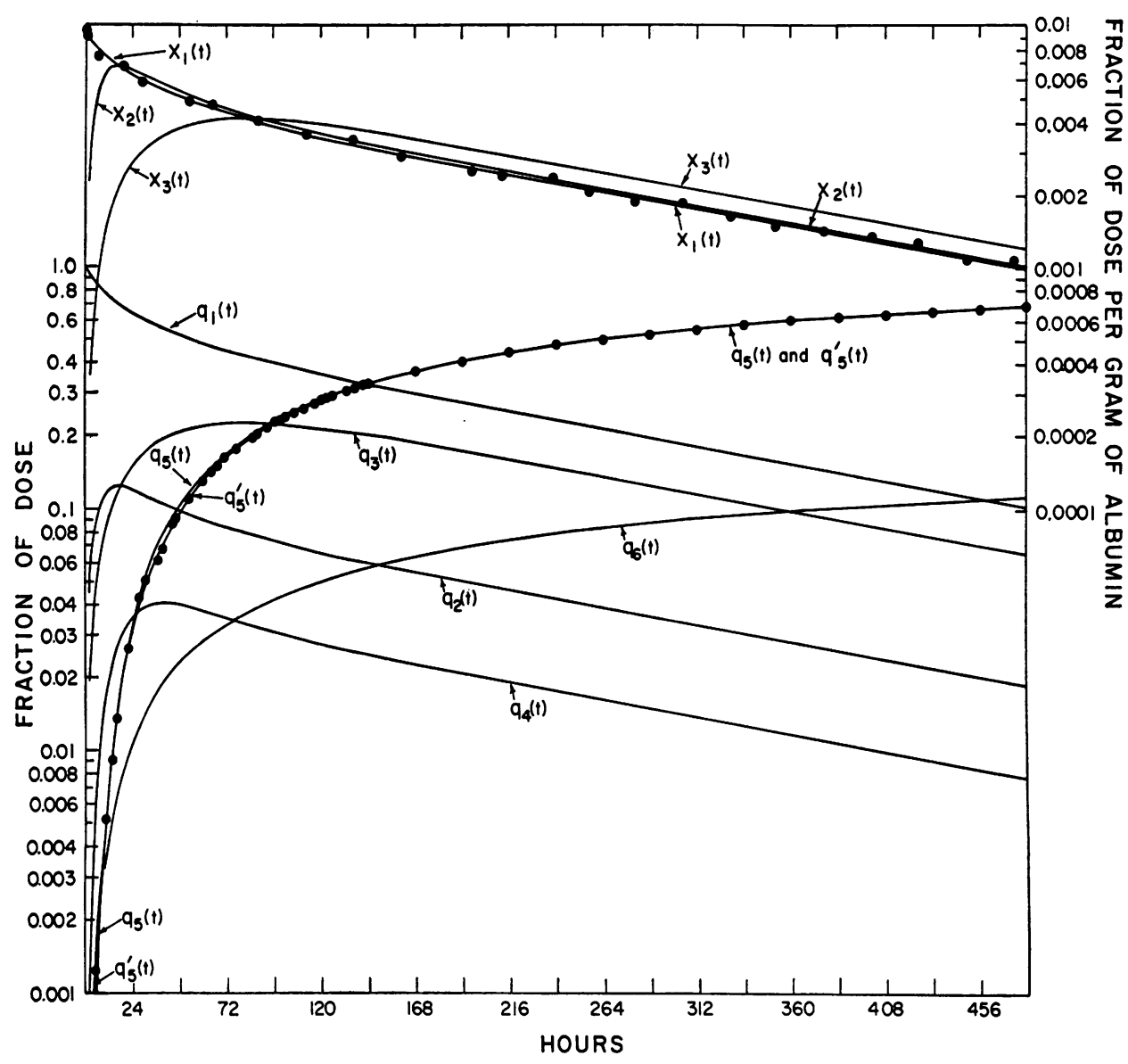

Fig. 8. Graphical Representation of the Open Parallel Solution

" $q$ " curves are plotted with reference to the left ordinate, " $x$ " curves with reference to the right ordinate. The curve labeled $q_{b}$ ' $(t)$ is the experimental urinary excretion curve. The curve labeled $q_{5}(t)$ represents the theoretical excretion curve for the model $\lambda_{23}=\lambda_{32}=0$ with Compartment 1 assumed to be the site of carrier entry. The solution parameters for this model appear in Tables I and II. The points on curve $x_{1}(t)$ are experimental. The curve as drawn is that reconstructed from the component values of $q_{1}(t)$ given in the text with appropriate normalization. 
large values of time is apparent. The degree to which the plotted experimental and theoretical excretion curves coincide is a measure of the closeness of fit of the experimental data to this model.

\section{DISCUSSION}

The analysis presented in this paper hinges predominantly on a system of three exchanging compartments. The additional compartments introduced (Compartments 4, 5 and 6) must be considered for quantitation of tracer within the exchanging system but do not influence the choice of the number of exchanging compartments. The latter is determined from the analysis of the plasma data alone.

Two independent sets of quantities may be distinguished in the general solution presented. One set consists of the fractional turnover rates of the compartments and these are determined from the tracer data alone. The second set consists of the amounts of carrier in each compartment and the rate of synthesis of carrier and its site of entry into the system. Specific activities are functions of both the tracer and carrier quantities.

Certain features of the solution presented (applicable to any three-compartment system for which data on Compartment 1 and the sum of Compartments 2 and 3 are available) are worth mentioning. The number of models which are mathematically compatible with the data and physically realizable is theoretically infinite, because any value of $\mathrm{x}$ and $\mathrm{y}$ in the shaded region of Figure 7 yields such a model. Despite this, a great deal can be said about the system. First, if one wishes to limit the solution to models that have the smallest number of interconnections, it is clear from Figure 7 that only four such models exist, the parallel model being one of these. The figure also indicates which models are not physically realizable. As noted above, the series model is excluded.

From Figure 7 it can be seen that (in addition to $\left.\lambda_{01}\right)$ at most four fractional turnover rates $\left(\lambda_{23}, \lambda_{31}, \lambda_{03}\right.$ and $\left.\lambda_{32}\right)$ may assume a value of zero, and that at most two of these may be zero in any single realizable model. Despite the infinity of realizable models, the values of the individual turnover rates may either be unique or vary over a limited range. Thus $\lambda_{11}, \lambda_{01}, \lambda_{21}+\lambda_{31}$ and $\lambda_{22}+\lambda_{33}$ are constant for all models. $\lambda_{22}, \lambda_{33}$ and $\lambda_{03}$ vary over very small ranges, whereas others show greater variation. Of particular interest is the fact that $\lambda_{02}$ is greater than zero in all realizable models and is consistently much larger than $\lambda_{03}$. This suggests that the rapidly exchanging extravascular compartment is the principal site of iodoalbumin degradation, a conclusion which is independent of the choice of model.

The values for the compartment sizes and rate of synthesis depend in part on the assumption of the site of entry of newly synthesized carrier. Assuming Compartment 1 to be the site of entry the solution for $S_{1}$ and for $S_{2}+S_{3}$ and consequently for $S_{1}+S_{2}+S_{3}$ is the same for all mathematically compatible models. Under the same conditions it is evident from Table II that $\rho_{s}$ is constant for all compatible models. For each different assumption concerning the site of entry, however, the values of $S_{2}+S_{3}, S_{1}+S_{2}+S_{3}$, and of $\rho_{\mathrm{s}}$ may be quite different. This is shown in Table II where solution values are given for three different sites of entry.

It is noteworthy that all the tracer data collected have been completely utilized in the solution presented. It is impossible therefore to reduce the uncertainty in the solution any further, regardless of the mathematical approach used, unless additional assumptions are made or new independent data be obtained.

In certain situations it may not be practical to apply routinely to tracer data a mathematical solution as general as the one presented here. One of the principal values of such a general approach, however, is that it formulates relationships inherent in the arbitrary assumption of model configurations which are sometimes not readily apparent. In addition it provides a way to evaluate the error in approximation methods and thereby serves to aid in the delineation of the conditions under which they are, or are not, acceptable.

In this connection it is of some interest to compare the values of certain parameters of the system obtained by the solution presented with the values obtained by approximation methods commonly employed. The "intercept" method is frequently used to compute total exchange- 
able carrier, $\mathrm{S}_{\mathrm{T}}$, from the equation:

21a) $\mathrm{S}_{\mathrm{T}}=\mathrm{S}_{1} / \mathrm{A}_{\text {ln }}$ or the equivalent expression

b) $\mathrm{S}_{\mathrm{T}}=1 / \mathrm{X}_{1 \mathrm{n}}$,

where $S_{1}$ is the size of the sampled compartment and $A_{1 n}$ and $X_{1 n}$ are the ordinate intercepts of the final exponential components of the radioactivity or specific activity, respectively, of the sampled compartment. Here $\mathrm{X}_{1 \mathrm{n}}$ is assumed to represent what the specific activity of the system as a whole would be at zero time if all the administered label were instantaneously and uniformly distributed throughout the system. Two sources of error are evident. 1) $\mathrm{x}_{1}$ for $\mathrm{t} \gg 0$ does not represent the mean specific activity of the system as a whole (see Equation 18). For the parallel model with entry of new carrier into Compartment 1 , for $t \gg 0$, the value of $\frac{x_{2}}{x_{1}}$ was $1.02, \frac{x_{3}}{x_{1}}$ was 1.21 , and $\frac{\mathrm{q}_{1}+\mathrm{q}_{2}+\mathrm{q}_{3}}{\mathrm{~S}_{1}+\mathrm{S}_{2}+\mathrm{S}_{3}} / \mathrm{x}_{1}$ was 1.07 . In general, the underestimation of the mean "equilibrium" specific activity of the system as a whole, if it be equated to that of the initially labeled compartment, leads to an overestimation of $S_{T}$ by Equation 21 . Other things being equal, the magnitude of the error is dependent on the relative magnitude of $\alpha_{3}$ compared to $\lambda_{22}$ and $\lambda_{33}$. 2) The intercept method assumes that the fractional rate of change of specific activity or radioactivity of the sampled compartment due to excretion of label from the system is constant throughout the experiment, and that $\frac{\mathrm{d}\left[\mathrm{q}_{1}(\mathrm{t})+\mathrm{q}_{2}(\mathrm{t})+\mathrm{q}_{3}(\mathrm{t})\right]}{\mathrm{dt}} /\left[\mathrm{q}_{1}(\mathrm{t})+\mathrm{q}_{2}(\mathrm{t})+\mathrm{q}_{3}(\mathrm{t})\right]=$ $\alpha_{\mathrm{n}}=$ constant, where $\mathrm{n}$ refers to the final component of the sampled compartment. This in turn assumes that $\lambda_{02}=\lambda_{03}=\lambda_{01}=\lambda_{0 \mathrm{~T}}$. Where inequalities of the rates of excretion from various compartments occur, this may lead to a greater or smaller fractional rate of excretion of total label in the early, as compared to the late, phases of the experiment, which will be reflected in smaller, or larger values, respectively, of $A_{1 n}$ and $X_{1 n}$. In general, where the fractional rate of excretion of total label in the early phase of the experiment exceeds that in the late phase, the effect is reflected in a smaller value of $X_{1 n}$, which increases the estimation of $S_{T}$ by Equation 21 . The converse also applies.
For the tracer data presented here, Equation 21 gave a value for $S_{T}$ of $195.3 \mathrm{Gm}$. as compared to $175.6 \mathrm{Gm}$. by three-compartment solution assuming new carrier entry into Compartment 1 . Experimental verification of some of the above considerations is found in the data of Gitlin, Janeway and Farr (14) who, in labeled iodoprotein studies in nephrosis, encountered measured values of edema fluid specific activity many times that of the plasma specific activity for large values of time and unreasonably large values of $S_{T}$ by the intercept method.

When total label in the exchanging system $\left(q_{\mathrm{T}}\right)$ can be computed from excretion data (with appropriate correction for ancillary compartments such as Compartments 4 and 6) $S_{T}$ is sometimes estimated by the "dilution" equation:

$$
\mathrm{S}_{\mathrm{T}}=\frac{\mathrm{q}_{\mathrm{T}}}{\mathrm{x}_{1}} \text { for } \mathrm{t} \gg 0 \text {. }
$$

The error in this method depends on the degree to which $\mathrm{x}_{1}$ differs from the mean specific activity of the system as a whole as discussed above.

In biological tracer work, the parameters of the systems studied which commonly receive greatest attention are $S_{T}, \rho_{\mathrm{s}}$ and $\lambda_{0 \mathrm{~T}}$. In a threecompartment open system,

$$
\lambda_{0 T} \equiv \frac{S_{1} \lambda_{01}+S_{2} \lambda_{02}+S_{3} \lambda_{03}}{S_{1}+S_{2}+S_{3}} .
$$

These three quantities are interrelated in the steady state:

$$
\rho_{\mathrm{s}}=\mathrm{S}_{\mathrm{T}} \lambda_{0 \mathrm{~T}} .
$$

The sources and magnitudes of the errors involved in estimating $S_{T}$ by "intercept" and "dilution" methods have been discussed above. The commonly employed "slope" method equates $\lambda_{0 \mathrm{~T}}$ to $\alpha_{\mathrm{n}}$ where $\mathrm{n}$, as before, refers to the final exponential component of the sampled compartment and takes on a value of 3 for a system of three exchanging compartments. Examination of Table II where values of $\lambda_{0 \mathrm{~T}}$ are given for the four limiting models and the generating model shows that $\alpha_{3}$ may underestimate or overestimate $\lambda_{0 \mathrm{~T}}$ depending on the choice of model configuration and site of new carrier entry. For assumed entry into Compartment $1, \lambda_{0 \mathrm{r}}$ is constant and greater than $\alpha_{3}$.

The turnover rates of body proteins are of 
considerable theoretical importance. The difficulties encountered in attempts to measure a plasma protein turnover rate have been discussed at length by Luck (15) and will not be reiterated here. A faster turnover rate for serum proteins determined by $\mathrm{I}^{131}$ labeling as compared to biosynthetic labels with $\mathrm{C}^{14}$ or $\mathrm{S}^{85}$ has been reported by several workers $(16,17)$. By jet iodination, however, McFarlane (18) has reported an $\mathrm{I}^{181}$ labeled albumin which appears to behave in an identical fashion to albumin biosynthetically labeled with $\mathrm{C}^{14}$. Furthermore, Berson, Yalow, Schreiber and Post (7) have described multiple components apparently metabolized at different rates in the same preparation of iodoalbumin. Yalow and Berson have also reported evidence that $I^{181}$ albumin of high specific activity may be damaged by self-irradiation to such an extent that its apparent rate of degradation is accelerated (19). Furthermore, it is apparently not clear, where discrepancies between the turnover rates determined by the different types of labels have been encountered, to what extent the discrepancy is attributable to reutilization of the $\mathrm{C}^{14}$ or $\mathrm{S}^{25}$ label. We are aware of the questionable validity of iodoalbumin as a label for endogenous albumin. Further discussion of this subject is not pertinent here. It must be emphasized that the derived rates of transport, degradation and "sequestration" are valid only insofar as the biological behavior of the iodoalbumin employed reflects the biological behavior of endogenous albumin, and this question would appear to be unsettled.

It is clear that little could be said about the distribution effect on observed degradation if a nonhomogeneous labeled material were used. The approach employed here of using the donor as a "biological filter" to screen out more rapidly degraded components would appear to be the method of choice at present to obtain material biologically homogeneous. The general inapplicability of this approach is obvious, however.

The use of a general kinetic formulation has brought to light in a formal way several aspects of iodoalbumin metabolism which apparently have not previously appeared in the literature: the quantitation of transcapillary transport rates of an open system, the probability of a very slowly exchanging or nonexchanging body com- partment, the favoring of the rapidly exchanging extravascular compartment as the principal site of iodoalbumin degradation, and kinetic evidence suggesting the physically realizable three-compartment series model to be incompatible with the experimental data.

Little can be said regarding the significance of the postulated Compartment 6 . There is experimental evidence that many of the plasma proteins do exist intracellularly in a variety of tissues where they maintain their identity at least to the extent of reacting with specific antibody (20). To our knowledge it has not been demonstrated that these intracellular proteins exchange with circulating plasma proteins. In addition it is known that antigens after injection may enter tissue cells where they may remain, apparently unchanged, for long periods of time. Since heavily iodinated albumin differs antigenically from native albumin (21) it is possible that the labeled protein entering Compartment 6 represents a heavily iodinated component in the injected material which is being treated by the body as an antigen.

The model employed here undoubtedly represents an over-simplification of the biological system. Compartments 2 and 3 probably each represent a composite of tissues having mean turnover rates of the approximate magnitude computed in the analysis. Although the actual biological system could undoubtedly be better represented by a more complex model, it is evident from the data that the system cannot be adequately described by a model of lesser complexity.

\section{SUMMARY}

Tracer experiments employing I ${ }^{181}$ labeled iodoalbumin in myxedematous and euthyroid patients indicated a kinetic system containing at least three exchanging compartments and three ancillary compartments (including urinary excretion). Additional studies indicated that the principal excretion forms were urinary iodide and diiodotyrosine. To insure a biologically homogeneous labeled material, one patient was studied in whom the administered labeled albumin was obtained from a donor patient after an eight-day period.

The analysis of the data from the latter tracer study is presented in some detail. The general 
solution of a three-compartment open system is given as well as modifications necessary for the solution of a six-compartment open system of the type encountered in the tracer studies. A mapping procedure preserving the invariants of the data was carried out and its value in delineating mathematically compatible and physically realizable models as well as the limits of computed turnover rates for nonunique solutions is illustrated. The numerical effect of variation of the site of entry of newly synthesized carrier into the system on various parameters of the solution is demonstrated.

The solution is further developed in such a way as to permit quantitation of inequalities of final specific activities in the compartments of an open system. Equations for the determination of errors in "intercept," "dilution" and "slope" methods are presented.

Computed values for asymptotic urinary excretion of label suggested the presence of a body compartment having a very slow turnover rate. The parallel model was shown to be one of an infinite number of mathematically compatible and physically realizable models, while the series model was excluded. The analysis suggested that Compartment 2 , the rapidly exchanging extravascular compartment, is the principal site of iodoalbumin degradation, irrespective of the model chosen.

\section{REFERENCES}

1. Berman, M., and Schoenfeld, R. Invariants in experimental data on linear kinetics and the formulation of models. J. appl. Physics 1956, 27, 1361.

2. Lewallen, C. G., Rall, J. E., and Berman, M. Studies of iodoalbumin metabolism. II. The effects of thyroid hormone. J. clin. Invest. 1959, 38, 88.

3. Siri, W. E. Isotopic Tracers and Nuclear Radiations. New York, McGraw-Hill Book Company, Inc., 1949, ch. XV.

4. Snedecor, G. W. Statistical Methods Applied to Experiments in Agriculture and Biology, 4th ed. Ames, Iowa, The Collegiate Press, 1946, ch. VI.

5. Sterling, $K$. The turnover rate of serum albumin in man as measured by I $^{181}$-tagged albumin. J. clin. Invest. 1951, 30, 1228.

6. Wasserman, K., and Mayerson, H. S. Exchange of albumin between plasma and lymph. Amer. J. Physiol. 1951, 165, 15.
7. Berson, A., Yalow, R. S., Schreiber, S. S., and Post, J. Tracer experiments with I'131-labeled human serum albumin: Distribution and degradation studies. J. clin. Invest. 1953, 32, 746.

8. Myant, N. B. Observations on the metabolism of human gamma globulin labeled by radioactive iodine. Clin. Sci. 1952, 11, 191.

9. Berson, S. A., and Yalow, R. S. The distribution of $I^{121}$ labeled human serum albumin introduced into ascitic fluid: Analysis of the kinetics of a three compartment catenary transfer system in man and speculation on possible sites of degradation. J. clin. Invest. 1954, 33, 377.

10. Albert, A., and Keating, F. R., Jr. Metabolic studies with ${ }^{131}$ labeled thyroid compounds: Distribution and excretion of radiodiiodotyrosine in human beings. J. clin. Endocr. 1951, 11, 996.

11. Skanse, B. Radioactive iodine in the diagnosis of thyroid disease. Acta med. scand. 1949, Suppl. 235, 1.

12. Riggs, D. S. Quantitative aspects of iodine metabolism in man. Pharmacol. Rev. 1952, 4, 284.

13. Keating, F. R., Jr., Power, M. H., Berkson, J., and Haines, S. F. The urinary excretion of radioiodine in various thyroid states. J. clin. Invest. 1947, 26, 1138.

14. Gitlin, D., Janeway, C. A., and Farr, L. E. Studies on the metabolism of plasma proteins in the nephrotic syndrome. I. Albumin, $\gamma$-globulin and iron-binding globulin. J. clin. Invest. 1956, 35, 44.

15. Luck, J. M. On the "turnover" of plasma proteins. Amer. J. Med. 1956, 20, 317.

16. Armstrong, S. H., Jr., McLeod, K., Wolter, J., and Kukral, J. The persistence in the blood of the radioactive label of albumin, gamma globulins, globulins of intermediate mobility studied with $S^{35}$ and paper electrophoresis: Methods and preliminary results. J. Lab. clin. Med. 1954, 43, 918.

17. Margen, S., and Tarver, $H$. Comparative studies on the turnover of serum albumin in normal human subjects. J. clin. Invest. 1956, 35, 1161.

18. McFarlane, A. S. Labelling of plasma proteins with radioactive iodine. Biochem. J. 1956, 62, 135.

19. Yalow, R. S., and Berson, S. A. Chemical and biological alterations induced by irradiation of $I^{12}$ labeled human serum albumin. J. clin. Invest. 1957, 36, 44.

20. Gitlin, D., Landing, B. H., and Whipple, A. The localization of homologous plasma proteins in the tissues of young human beings as demonstrated with fluorescent antibodies. J. exp. Med. 1953, 97, 163.

21. Friedberg, W., Walter, H., and Haurowitz, F. The fate in rats of internally and externally labelled heterologous proteins. J. Immunol. 1955, 75, 366. 